Document downloaded from:

http://hdl.handle.net/10251/105468

This paper must be cited as:

Vila Tortosa, MP.; Fayos Sancho, J.; Baeza González, LM. (2011). Simulation of the evolution of rail corrugation using a rotating flexible wheelset model. Vehicle System Dynamics. 49(11):1749-1769. doi:10.1080/00423114.2011.552619

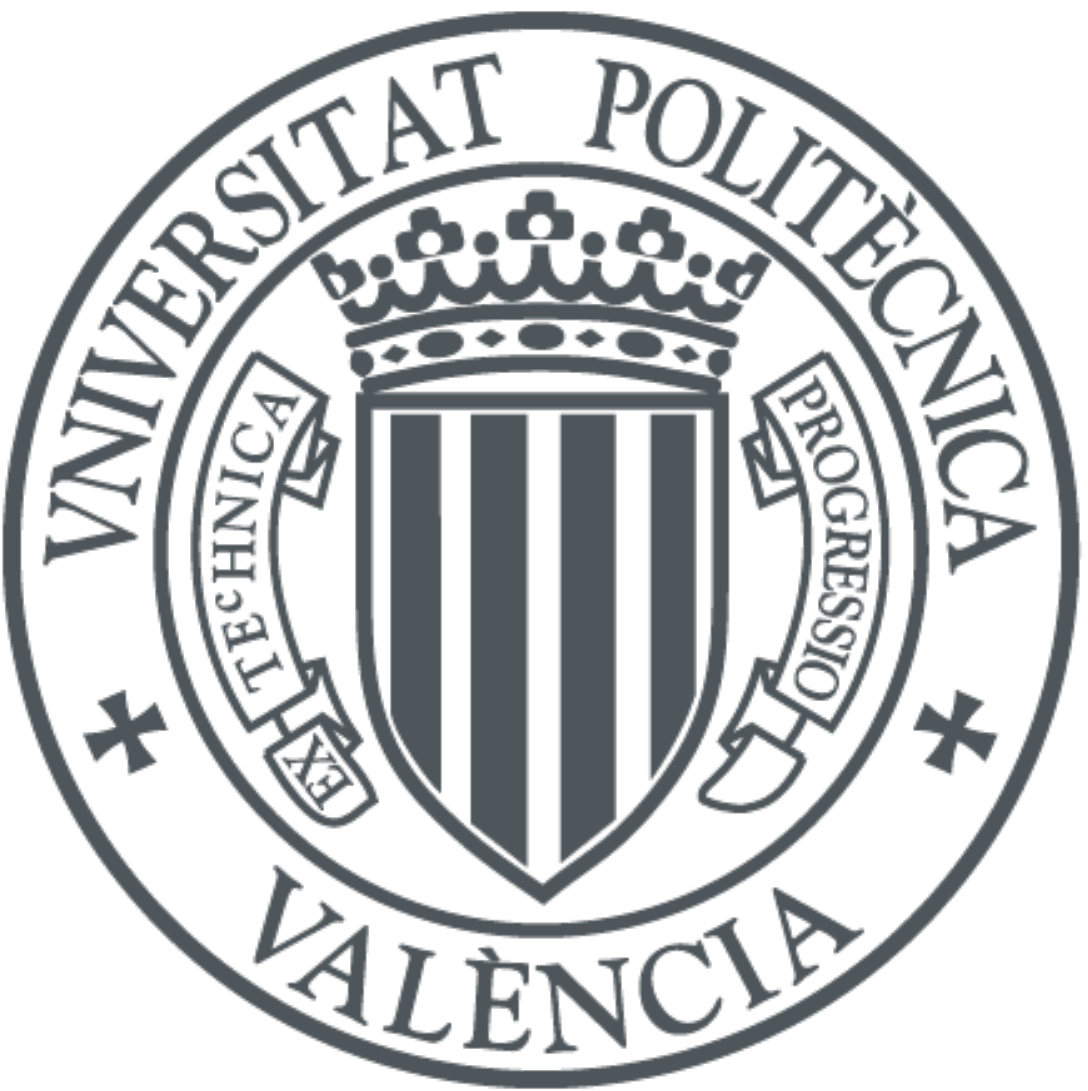

The final publication is available at

http://dx.doi.org/10.1080/00423114.2011.552619

Copyright TAYLOR \& FRANCIS LTD

Additional Information 


\title{
Simulation of the evolution of rail corrugation by using a rotating flexible wheelset model
}

\author{
Paloma Vila ${ }^{(1)}$, Juan Fayos ${ }^{(2)}$ and Luis Baeza ${ }^{(1) *}$ \\ ${ }^{(1)}$ Centro de Investigación en Tecnología de Vehículos, Universitat Politècnica de València, \\ Camino de Vera s/n, 46022 Valencia, Spain \\ ${ }^{(2)}$ Instituto de Biomecánica de Valencia, Universitat Politècnica de València, Camino de Vera \\ $s / n, 46022$ Valencia, Spain
}

*Corresponding author. E-mail: lbaeza@mcm.upv.es

\begin{abstract}
This paper presents a simulation tool designed for predicting the wear pattern on the running surface of the rails, and for studying the evolution of rail corrugation after thousands of wheelset passages. This simulation tool implements a cyclic track model, a rotating flexible wheelset model, a wheel-rail contact model and a wear model. The vehicle-track system is modelled by using a substructuring technique, by which the vehicle, the rails, the sleepers are treated independently of each other and are coupled by the forces transmitted through the wheel-rail contact and the railpad. The vehicle model takes only account of the wheelset since the sprung masses of the vehicle are not relevant in the frequency range analysed. The wheelset model considers the flexibility of the wheelset and the effects associated with rotation. By using the Campbell diagram, two cases have been identified in which the combined effect of two different modes may give rise to higher wheel-rail contact forces and wear.
\end{abstract}

Keywords: rail corrugation, cyclic track, rotating flexible wheelset, substructuring technique.

\section{Introduction}

Corrugation in rails remains nowadays one of the most widely spread defects in tracks worldwide. This defect is known not only to give rise to high-frequency vibrations, noise nuisance and loss of ride comfort, but also to trigger the formation of cracks in rails. Until now, rail grinding and friction modifiers are the only techniques that have proved to be effective in controlling corrugation. But, at the same time, the former technique leads to notably higher maintenance costs to be faced by railway administrations.

Much research efforts have focused on the understanding of the initiation and further development of rail corrugation with the aim to prevent or at least control the formation of this defect. However, since the first reports on corrugation at the end of the 19th century [1], only a few corrugation types were explained through mathematical models (see an example in [2]). Most of the wavelength-fixing mechanisms (see definition in [3]) were broadly found experimentally by identifying the corresponding frequency of the corrugation with certain natural frequency or resonance of the vehicle unsprung mass-track system [4]. The corrugation is understood, then, as a dynamic problem associated with wear and/or plastic deformation in the wheel/rail contact patch. Consequently, any dynamic amplification of the train-track system can potentially be a wavelength-fixing mechanism. If the dynamics of the system behaves linearly (which is a coherent hypothesis, at least in the beginning stages), the corrugation should be 
considered as a fixed-frequency phenomenon with few exceptions. One special case may be the dynamics of the rotating wheelset, where its resonance frequencies depend on the angular velocity of the wheelset.

Classic works on Jeffcott rotors and rotating beams showed that the equivalent vibration modes of the shaft change with the angular velocity (see monograph in [5]). Each mode with multiplicity 2 of the non-rotating system splits into two different modes: the forward mode, whose deformed shape rotates in the same direction of the spin motion and the backward mode, in the opposite direction. The difference between the frequencies of the backward and forward modes of a simply supported Rayleigh beam was obtained in [6], that is

$$
\Delta \omega_{k}=\frac{2 k^{2} \pi^{2}}{k^{2} \pi^{2}+\lambda^{2}} \Omega
$$

where $k$ is the mode index, $\Omega$ is the spinning angular velocity and $\lambda$ is the beam slenderness. If the slenderness is zero (the railway wheelset slenderness should be small) the difference between the modes is twice the spinning velocity.

The literature showed few advanced models of the railway wheelset. A flexible but non-rotating wheelset model was published in [7], and the moving load effect associated with the rotation of a flexible wheel was considered in [8]. It was stated that a commercial simulation package implemented a flexible and rotating wheelset model but the method is unpublished and cannot be reproduced. The work in [9] presented a method for obtaining the dynamic response of rotating flexible solids. This model takes account of the inertial and the moving load effects due to rotation. The technique was applied to the railway case in [10].

The objective of the present work is to demonstrate the capability of the wheelset dynamics to produce corrugation in rails. To this end, a computational tool that permits to simulate the evolution of the rail roughness has been developed. The program combines a dynamic model of the wheelset-track system and a wear model, in accordance with the sketch of Figure 1. The track model is based on the periodic vehicle-track system proposed in [11]. The track model considers the rail torsion, the rotation of the rail section and the lateral and vertical deflections of the rail. The FASTSIM algorithm is adopted to compute the contact forces and the local slip.

The program implements the wheelset model developed in [10]. This wheelset model takes account of the inertial effects due to rotation, that is, it is a rotating wheelset model, in contrast to those wheelset models that do not consider these effects, which are known as non-rotating wheelset models. A new procedure for developing the wheelset model can be found in Section 2 of the present article. Section 3 presents the influence of the angular velocity of the wheelset on its natural frequencies. Finally, in Section 4 results from simulation, namely contact forces and wear, are shown. 


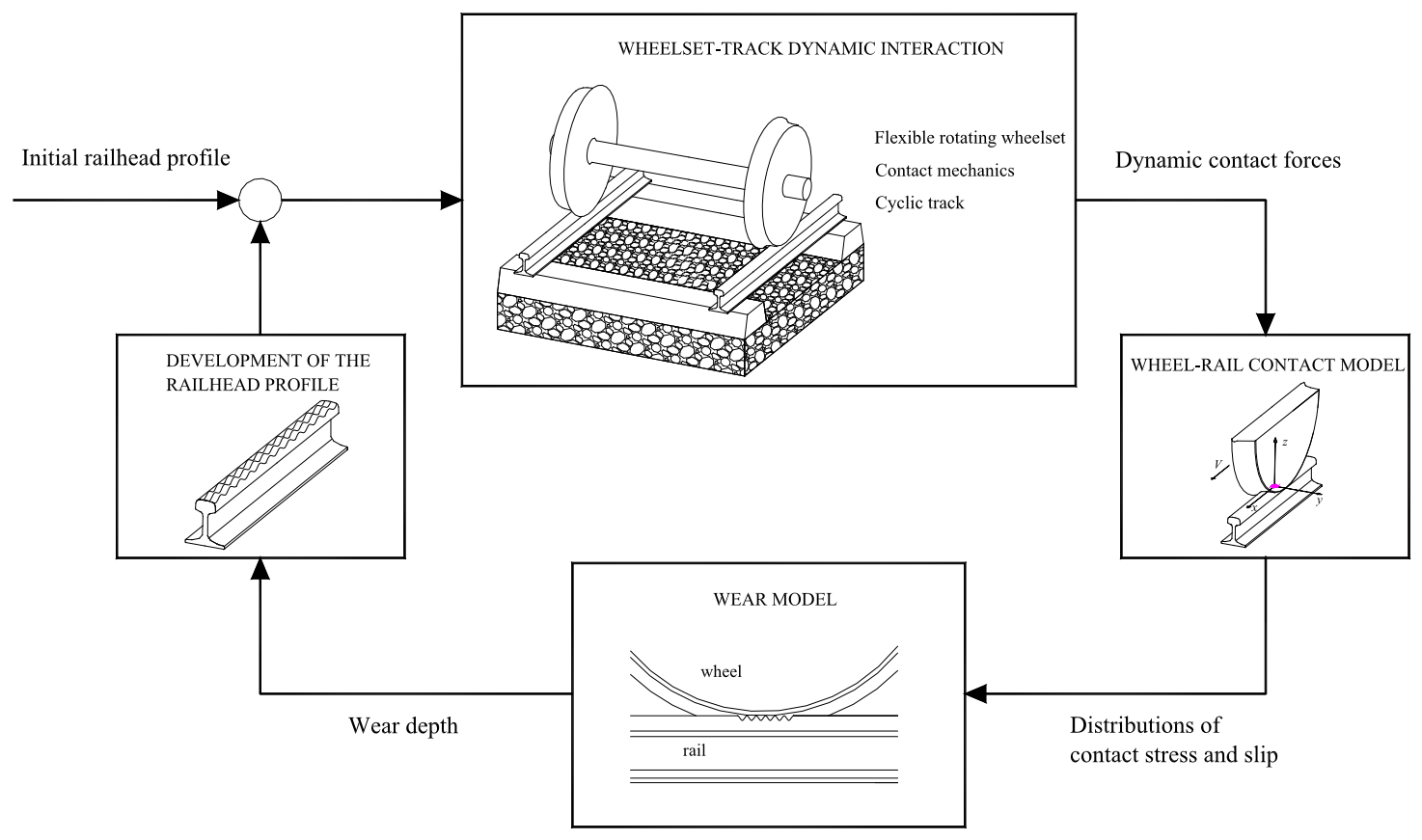

Figure 1. Structure of the simulation tool (following the scheme proposed in [3]).

\section{Description of the simulation tool}

The simulation tool developed in this work is intended to estimate the wear on the running surface of the rails and to study the evolution of rail corrugation. To this aim, the simulation tool implements a cyclic track model, a rotating flexible wheelset model, a wheel-rail contact model and a wear theory. The modelling of the system dynamics is done through a substructuring technique, in which the vehicle, the rails and each sleeper are considered in an isolated way. The coupling between the substructures is carried out through the wheel-rail contact forces and the forces transmitted through the railpads. The former forces are calculated by adopting a theory of contact, FASTSIM in this case, and the latter forces are computed through the relative displacements between the sleepers and the rails. Regarding the model of the vehicle, only a wheelset is considered since the influence of the sprung masses of the vehicle is negligible in the frequency range to study. Tables 1 and 2 contain the values of the significant parameters of the different substructures constituting the model of the track and the parameters of the wheelset model, respectively. Next, the models of the track and the wheelset are described. 
Table 1. Parameters of the track model.

\begin{tabular}{ll}
\hline Rail & \\
Density of the rail & $7850 \mathrm{~kg} / \mathrm{m}^{3}$ \\
Young's modulus of the rail & $2.1 \times 10^{11} \mathrm{~N} / \mathrm{m}^{2}$ \\
Shear modulus of the rail & $0.81 \times 10^{11} \mathrm{~N} / \mathrm{m}^{2}$ \\
Cross-sectional area of the rail & $7.69 \times 10^{-3} \mathrm{~m}^{2}$ \\
Second moment of area of the rail cross-section & $30.55 \times 10^{-6} \mathrm{~m}^{4}$ \\
Shear coefficient of the rail cross-section & 0.4 \\
Railpad & \\
Vertical rail pad stiffness & $3.5 \times 10^{8} \mathrm{~N} / \mathrm{m}$ \\
Rail pad damping coefficient & $50 \times 10^{3} \mathrm{Ns} / \mathrm{m}$ \\
Sleeper & \\
Mass of the sleeper & $324 \mathrm{~kg}$ \\
Sleeper spacing & $0.6 \mathrm{~m}$ \\
Ballast & \\
Vertical ballast stiffness & $100 \times 10^{6} \mathrm{~N} / \mathrm{m}$ \\
Damping coefficient of ballast & $200 \times 10^{3} \mathrm{Ns} / \mathrm{m}$ \\
Friction coefficient & 0.3 \\
\hline
\end{tabular}

Table 2. Parameters of the wheelset model.

\begin{tabular}{ll}
\hline Wheelset properties & \\
\hline Mass & $2700 \mathrm{~kg}$ \\
Static load & $200 \mathrm{kN}$ \\
Rolling radius & $0.5 \mathrm{~m}$ \\
Moment in the vertical and lateral axes & $493 \mathrm{~kg} \mathrm{~m}^{2}$ \\
Moment in the rolling axis & $169 \mathrm{~kg} \mathrm{~m}^{2}$ \\
\hline
\end{tabular}

\subsection{Track model}

In the literature, it can be found both track models representing a finite-length stretch of the track and track models of infinite length. Most of the models of the former group are based on the direct application of the Finite Element Method, which on the one hand, represent the track in a realistic way but, on the other hand, are inefficient from a computational viewpoint. All the finite-length track models present a border effect, which causes the waves to reflect back at the end of the model. Thus, the waves interact again with the vehicle, spoiling the results from the simulation. This fact forces to adopt large lengths in order to minimise the border effect, especially when the simulation time and the velocity of the vehicle are high.

Among the most mathematically sophisticated models are the infinite track models, in which the rails are assumed to be discretely supported by the sleepers. There are two important groups of infinite track models: the first group uses the Fourier transform in the frequency and time domain [12] and the second group is based on the wave 
propagation models in periodic structures [13]. Both groups of track models require the assumption of hypotheses of linearity for the mechanical properties of the track.

An alternative that maintains the versatility of the finite track models and minimises the border effect corresponds to [14]. In this work, a cyclic vehicle-track model is presented, which consists in an infinite track on which an infinite number of identical vehicles circulate. The distance between two consecutive vehicles is chosen to be the characteristic length $L$, as can be seen in Figure 2. The modelling of the track is done only for a stretch of the track with the characteristic length $L$. The adoption of a cyclic track model has the advantage, thanks to the periodicity properties, that a finite track length can be considered to analyse an infinite track without affecting the computing accuracy. Thus, the boundary conditions at both ends of the finite track are the same and the infinite track can be modelled through the modal properties of the stretch by employing a substructuring technique. The track model consists of several substructures: rails, railpads, sleepers and ballast. Each track substructure is related to a set of modal equations. The rails and sleepers are modelled as flexible beams. The rails are considered as cyclic Timoshenko beams, whose mode shapes can be calculated analytically.

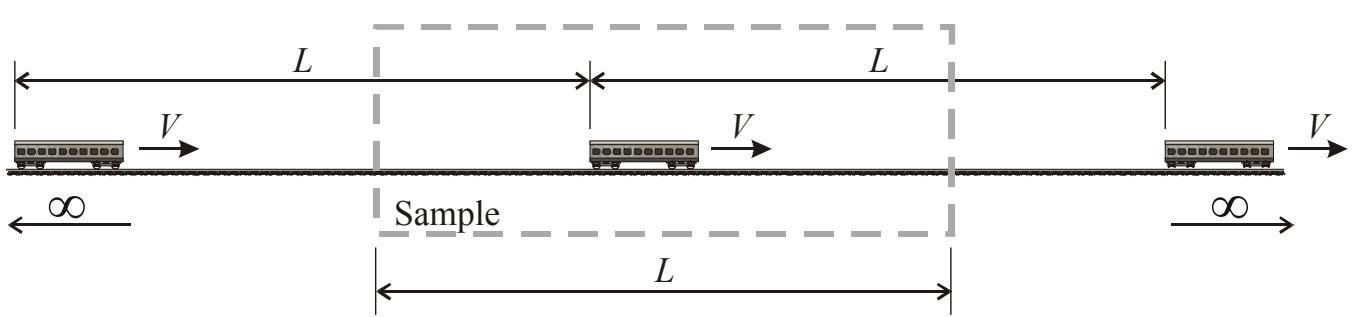

Figure 2. Sketch of a cyclic vehicle-track model.

\subsection{Wheelset model}

\subsubsection{The Lagrangian method}

The coordinate system adopted for the modelling of the rotating flexible wheelset model is based on the formulation of floating frame of reference proposed in [14]. This method was adopted in [15] in order to develop the equations of motion of a rotating solid. This method obtains the global position of a flexible solid as a sum of two different displacement types. The former can be considered as a rigid body displacement, the latter corresponds to the displacements due to the deformation of the solid.

The floating frame method makes use of two reference frame systems: an inertial frame and a mobile frame, which rotates at the angular velocity of the solid $\Omega$ and it is associated with the undeformed configuration. A vector referred to the fixed and the mobile frames are denoted by $\mathbf{a}$ and $\overline{\mathbf{a}}$, respectively. The vector position $\mathbf{r}$ of a material particle of the solid in relation to the origin of the fixed frame can be expressed as follows

$$
\mathbf{r}=\mathbf{A}(\overline{\mathbf{u}}+\boldsymbol{\Phi}(\overline{\mathbf{u}}) \mathbf{p}(t)),
$$


where $\overline{\mathbf{u}}$ is the vector position of the particle in the undeformed configuration in the mobile frame. The product $\boldsymbol{\Phi}(\overline{\mathbf{u}}) \mathbf{p}(t)$ corresponds to the displacement of the particle due to the elastic deformation of the solid in the rotating frame, being $\boldsymbol{\Phi}(\overline{\mathbf{u}})$ the matrix of functions of the mass-normalised vibration modes of the non-rotating structure, and $\mathbf{p}(t)$ is the modal coordinate; $\mathbf{A}$ is the following rotation matrix

$$
\mathbf{A}=\left(\begin{array}{ccc}
\cos \theta & 0 & \sin \theta \\
0 & 1 & 0 \\
-\sin \theta & 0 & \cos \theta
\end{array}\right)
$$

with $\theta=\Omega t$ being the angle of rotation.

The method presented in [16] develops the following equation of motion for rotating solids

$$
\ddot{\mathbf{p}}+2 \Omega \widetilde{\mathbf{J}} \dot{\mathbf{p}}+\left(\widetilde{\mathbf{K}}-\Omega^{2} \widetilde{\mathbf{E}}\right) \mathbf{p}=\mathbf{Q}_{\mathbf{p}}+\Omega^{2} \widetilde{\mathbf{L}}
$$

The components of the last equation are presented as follows. Matrix $\widetilde{\mathbf{K}}$ is the modal stiffness matrix whose diagonal contains the square of the undamped natural frequencies of the solid. Matrix $\widetilde{\mathbf{J}}$ is associated with the gyroscopic effect. It is computed as follows

$$
\widetilde{\mathbf{J}}=\int_{\text {Volume }} \rho \boldsymbol{\Phi}^{\mathrm{T}} \mathbf{J} \boldsymbol{\Phi} \mathrm{d} v
$$

where

$$
\mathbf{J}=\mathbf{A}_{\theta} \mathbf{A}^{\mathrm{T}}=\mathbf{A}^{\mathrm{T}} \mathbf{A}_{\theta},
$$

with $\mathbf{A}_{\theta}$ being the derivative of the matrix $\mathbf{A}$ with respect to $\theta$. The development of the Equation (6) shows that

$$
\mathbf{J}=\left(\begin{array}{ccc}
0 & 0 & 1 \\
0 & 0 & 0 \\
-1 & 0 & 0
\end{array}\right)
$$

Some vibration modes produce deformed shapes which may cause centrifugal forces to produce work. This effect is considered through the matrix $\widetilde{\mathbf{E}}$, which is calculated as follows

$$
\widetilde{\mathbf{E}}=\int_{\text {Volume }} \rho \boldsymbol{\Phi}^{\mathrm{T}} \mathbf{E} \boldsymbol{\Phi} \mathrm{d} v
$$

where

$$
\mathbf{E}=-\mathbf{A}_{\theta \theta} \mathbf{A}^{\mathrm{T}}=-\mathbf{A}^{\mathrm{T}} \mathbf{A}_{\theta \theta},
$$


with $\mathbf{A}_{\theta \theta}$ being the second derivative of the matrix $\mathbf{A}$ with respect to $\theta$. It can be easily obtained that

$$
\mathbf{E}=\left(\begin{array}{lll}
1 & 0 & 0 \\
0 & 0 & 0 \\
0 & 0 & 1
\end{array}\right)
$$

The centrifugal forces that do not depend on the solid deformation are implemented by means of $\widetilde{\mathbf{L}}$. These forces are associated with modes of single multiplicity, and the entries of $\widetilde{\mathbf{L}}$ are non-zero only associated with modes of single multiplicity. The column matrix $\widetilde{\mathbf{L}}$ is obtained as follows

$$
\widetilde{\mathbf{L}}=\int_{\text {Volume }} \rho \boldsymbol{\Phi}^{\mathrm{T}} \mathbf{E} \overline{\mathbf{u}} \mathrm{d} v
$$

If the external forces are applied in fixed material points, the generalised force term is computed as follows

$$
\mathbf{Q}_{\mathbf{p}}(t)=\int_{\text {Volume }} \boldsymbol{\Phi}(\overline{\mathbf{u}})^{\mathrm{T}} \mathbf{A}(t)^{\mathrm{T}} \mathbf{f}^{v}(\overline{\mathbf{u}}, t) \mathrm{d} v
$$

where $\mathbf{f}^{v}(\overline{\mathbf{u}}, t)$ is the volume external forces. It can be pointed out that the integral kernel is function of time.

\subsubsection{The Eulerian approach}

The properties of the solids of revolution allow defining the deformed shape of the solid through the modal functions in non-rotating coordinates as follows. Let $\mathbf{v}$ be the vector position of a spatial point in relation to the origin of the fixed frame. The vector $\mathbf{v}$ is defined in the undeformed domain or the volume occupied by the undeformed configuration. The vector position of a particle that occupies the spatial position $\mathbf{v}$ in the undeformed configuration at the instant $t$ verifies

$$
\mathbf{v}=\mathbf{A} \overline{\mathbf{u}} .
$$

The position of the particle after deformation is

$$
\mathbf{r}=\mathbf{v}+\boldsymbol{\Phi}(\mathbf{v}) \mathbf{q}(t)
$$

if the particle occupies the spatial position $\mathbf{v}$ in the undeformed configuration, with $\mathbf{q}(t)$ being the Eulerian-modal coordinate vector. From Equations (2) and (14), it follows that

$$
\boldsymbol{\Phi}(\mathbf{v}) \mathbf{q}(t)=\mathbf{A} \boldsymbol{\Phi}(\overline{\mathbf{u}}) \mathbf{p}(t)
$$


Equation (15) is multiplied by $\rho \boldsymbol{\Phi}(\mathbf{v})^{\mathrm{T}}$, where $\rho$ is the material density of the solid, and then it is integrated over the volume of the solid, that is

$$
\mathbf{q}(t)=\left(\int_{\text {Volume }} \rho \boldsymbol{\Phi}(\mathbf{v})^{\mathrm{T}} \mathbf{A} \boldsymbol{\Phi}\left(\mathbf{A}^{\mathrm{T}} \mathbf{v}\right) \mathrm{d} v\right) \mathbf{p}(t)
$$

The last equation provides the coordinate transformation, which can be written in the following manner

$$
\mathbf{q}(t)=\mathbf{B}(t) \mathbf{p}(t)
$$

in which $\mathbf{B}(t)$ is the transformation matrix that can be proved to be orthogonal (Appendix C) and quasi-diagonal (Appendix D).

The coordinate change in Equation (17) is carried out in Equation (4), and then the resulting equation is multiplied by $\mathbf{B}(t)$, resulting

$$
\begin{aligned}
& \ddot{\mathbf{q}}+\left(2 \Omega \mathbf{B} \widetilde{\mathbf{J}} \mathbf{B}^{\mathrm{T}}+2 \mathbf{B} \dot{\mathbf{B}}^{\mathrm{T}}\right) \dot{\mathbf{q}}+ \\
& +\left(\mathbf{B} \widetilde{\mathbf{K}} \mathbf{B}^{\mathrm{T}}-\Omega^{2} \mathbf{B} \widetilde{\mathbf{E}} \mathbf{B}^{\mathrm{T}}+\mathbf{B} \ddot{\mathbf{B}}^{\mathrm{T}}+2 \Omega \mathbf{B} \widetilde{\mathbf{J}} \dot{\mathbf{B}}^{\mathrm{T}}\right) \mathbf{q}=\mathbf{B} \mathbf{Q}_{\mathbf{p}}+\Omega^{2} \mathbf{B} \widetilde{\mathbf{L}}
\end{aligned}
$$

Taking into account the properties stated in the Appendixes A, B and E, the following equations are found

$$
\begin{aligned}
\mathbf{B} \widetilde{\mathbf{J}} \mathbf{B}^{\mathrm{T}} & =\widetilde{\mathbf{J}} \\
\mathbf{B} \widetilde{\mathbf{E}} \mathbf{B}^{\mathrm{T}} & =\widetilde{\mathbf{E}} \\
\mathbf{B} \widetilde{\mathbf{K}} \mathbf{B}^{\mathrm{T}} & =\widetilde{\mathbf{K}} \\
\text { and } \mathbf{B} \widetilde{\mathbf{L}} & =\widetilde{\mathbf{L}}
\end{aligned}
$$

The calculation of the term $\mathbf{B} \widetilde{\mathbf{J}} \dot{\mathbf{B}}^{\mathrm{T}}$ is reduced to the resolution of $\mathbf{B} \dot{\mathbf{B}}^{\mathrm{T}}$, because $\mathbf{B} \widetilde{\mathbf{J}}=\mathbf{B} \widetilde{\mathbf{J}} \mathbf{B}^{\mathrm{T}} \mathbf{B}=\widetilde{\mathbf{J}} \mathbf{B}$. From Equation (C.2),

$$
\mathbf{B} \boldsymbol{\Phi}(\overline{\mathbf{u}})^{\mathrm{T}}=\boldsymbol{\Phi}(\mathbf{v})^{\mathrm{T}} \mathbf{A}
$$

The last equation is derived with respect to time,

$$
\dot{\mathbf{B}} \boldsymbol{\Phi}(\overline{\mathbf{u}})^{\mathrm{T}}=\dot{\boldsymbol{\Phi}}(\mathbf{v})^{\mathrm{T}} \mathbf{A}+\boldsymbol{\Phi}(\mathbf{v})^{\mathrm{T}} \dot{\mathbf{A}}
$$

In the last equation the function $\boldsymbol{\Phi}(\overline{\mathbf{u}})$ is constant since it is associated with a material point. On the other hand, $\dot{\Phi}(\mathbf{v})$ has to be computed as the convective term of the material derivative, that is 


$$
\begin{aligned}
\dot{\boldsymbol{\Phi}}(\mathbf{v}) & =\sum_{i=1}^{3} \frac{\partial \boldsymbol{\Phi}(\mathbf{v})}{\partial v_{i}} \frac{\mathrm{d} v_{i}}{\mathrm{~d} t}=\sum_{i=1}^{3} \frac{\partial \boldsymbol{\Phi}(\mathbf{v})}{\partial v_{i}} \frac{\mathrm{d}(\mathbf{A} \overline{\mathbf{u}})_{i}}{\mathrm{~d} t} \\
& =\sum_{i=1}^{3} \frac{\partial \boldsymbol{\Phi}(\mathbf{v})}{\partial v_{i}}\left(\Omega \mathbf{A}_{\theta} \overline{\mathbf{u}}\right)_{i}=\Omega \sum_{i=1}^{3} \frac{\partial \boldsymbol{\Phi}(\mathbf{v})}{\partial v_{i}}(\mathbf{J} \mathbf{v})_{i}
\end{aligned}
$$

Equation (25) is now post-multiplied by $\boldsymbol{\Phi}(\overline{\mathbf{u}}) \mathbf{B}^{\mathrm{T}}$ resulting

$$
\dot{\mathbf{B}} \boldsymbol{\Phi}(\overline{\mathbf{u}})^{\mathrm{T}} \boldsymbol{\Phi}(\overline{\mathbf{u}}) \mathbf{B}^{\mathrm{T}}=\Omega\left(\sum_{i=1}^{3} \frac{\partial \boldsymbol{\Phi}(\mathbf{v})^{\mathrm{T}}}{\partial v_{i}}(\mathbf{J} \mathbf{v})_{i}\right) \mathbf{A} \boldsymbol{\Phi}(\overline{\mathbf{u}}) \mathbf{B}^{\mathrm{T}}+\Omega \boldsymbol{\Phi}(\mathbf{v})^{\mathrm{T}} \mathbf{A}_{\theta} \boldsymbol{\Phi}(\overline{\mathbf{u}}) \mathbf{B}^{\mathrm{T}}
$$

Recalling the result in Equation (14), the last equation becomes

$$
\begin{aligned}
\dot{\mathbf{B}} \boldsymbol{\Phi}(\overline{\mathbf{u}})^{\mathrm{T}} \boldsymbol{\Phi}(\overline{\mathbf{u}}) \mathbf{B}^{\mathrm{T}} & =\Omega\left(\sum_{i=1}^{3} \frac{\partial \boldsymbol{\Phi}(\mathbf{v})^{\mathrm{T}}}{\partial v_{i}}(\mathbf{J} \mathbf{v})_{i}\right) \boldsymbol{\Phi}(\mathbf{v}) \mathbf{B} \mathbf{B}^{\mathrm{T}}+\Omega \boldsymbol{\Phi}(\mathbf{v})^{\mathrm{T}} \mathbf{A}_{\theta} \mathbf{A}^{\mathrm{T}} \boldsymbol{\Phi}(\mathbf{v}) \mathbf{B} \mathbf{B}^{\mathrm{T}} \\
& =\Omega\left(\sum_{i=1}^{3} \frac{\partial \boldsymbol{\Phi}(\mathbf{v})^{\mathrm{T}}}{\partial v_{i}}(\mathbf{J} \mathbf{v})_{i}\right) \boldsymbol{\Phi}(\mathbf{v})+\Omega \boldsymbol{\Phi}(\mathbf{v})^{\mathrm{T}} \mathbf{J} \boldsymbol{\Phi}(\mathbf{v})
\end{aligned}
$$

Now Equation (27) is multiplied by the density $\rho$ and it is integrated over the volume of the solid. By applying the property of orthogonality of the modes, it yields the following expression

$$
\dot{\mathbf{B}} \mathbf{B}^{\mathrm{T}}=\Omega \int_{\text {Volume }}\left(\sum_{i=1}^{3} \frac{\partial \boldsymbol{\Phi}(\mathbf{v})^{\mathrm{T}}}{\partial v_{i}}(\mathbf{J} \mathbf{v})_{i}\right) \boldsymbol{\Phi}(\mathbf{v}) \rho \mathrm{d} v+\Omega \widetilde{\mathbf{J}}
$$

Applying the first property in Appendix $\mathrm{C}$ the integral in the Equation (28) does not depend on time. It is associated with a new variable, that is

$$
\begin{gathered}
\widetilde{\mathbf{G}}=\int_{\text {Volume }}\left(\sum_{i=1}^{3} \frac{\partial \boldsymbol{\Phi}(\overline{\mathbf{u}})^{\mathrm{T}}}{\partial u_{i}}(\mathbf{J} \overline{\mathbf{u}})_{i}\right) \boldsymbol{\Phi}(\overline{\mathbf{u}}) \rho \mathrm{d} v \text {, and } \\
\dot{\mathbf{B}} \mathbf{B}^{\mathrm{T}}=\Omega(\widetilde{\mathbf{G}}+\widetilde{\mathbf{J}})
\end{gathered}
$$

The calculation of $\mathbf{B} \ddot{\mathbf{B}}^{\mathrm{T}}$ is performed as follows. The product $\dot{\mathbf{B}} \mathbf{B}^{\mathrm{T}}$ is constant and consequently

$$
0=\frac{\mathrm{d}}{\mathrm{d} t} \dot{\mathbf{B}} \mathbf{B}^{\mathrm{T}}=\ddot{\mathbf{B}} \mathbf{B}^{\mathrm{T}}+\dot{\mathbf{B}} \dot{\mathbf{B}}^{\mathrm{T}}
$$

From Equations (30) and (31), it is deduced

$$
\ddot{\mathbf{B}} \mathbf{B}^{\mathrm{T}}=-\dot{\mathbf{B}} \dot{\mathbf{B}}^{\mathrm{T}}=-\dot{\mathbf{B}} \mathbf{B}^{\mathrm{T}} \mathbf{B} \dot{\mathbf{B}}^{\mathrm{T}}=-\Omega^{2}(\widetilde{\mathbf{G}}+\widetilde{\mathbf{J}})(\widetilde{\mathbf{G}}+\widetilde{\mathbf{J}})^{\mathrm{T}}
$$

Further analysis will show that $\widetilde{\mathbf{G}}$ is antisymmetric, and the last expression can be written as follows 


$$
\ddot{\mathbf{B}} \mathbf{B}^{\mathrm{T}}=-\dot{\mathbf{B}} \dot{\mathbf{B}}^{\mathrm{T}}=-\dot{\mathbf{B}} \mathbf{B}^{\mathrm{T}} \mathbf{B} \dot{\mathbf{B}}^{\mathrm{T}}=\Omega^{2}(\widetilde{\mathbf{G}}+\widetilde{\mathbf{J}})(\widetilde{\mathbf{G}}+\widetilde{\mathbf{J}})
$$

The generalised force of the Equation (18) is, from (12)

$$
\mathbf{Q}_{\mathbf{q}}(t)=\mathbf{B} \mathbf{Q}_{\mathbf{p}}(t)=\mathbf{B} \int_{\text {Volume }} \boldsymbol{\Phi}\left(\mathbf{A}(t)^{\mathrm{T}} \mathbf{v}\right)^{\mathrm{T}} \mathbf{A}(t)^{\mathrm{T}} \mathbf{f}^{v}(\mathbf{v}, t) \mathrm{d} v
$$

By substituting Equation (C.2) into the last result, it is found

$$
\mathbf{Q}_{\mathbf{q}}(t)=\mathbf{B} \int_{\text {Volume }} \mathbf{B}^{\mathrm{T}} \boldsymbol{\Phi}(\mathbf{v})^{\mathrm{T}} \mathbf{f}^{v}(\mathbf{v}, t) \mathrm{d} v=\int_{\text {Volume }} \boldsymbol{\Phi}(\mathbf{v})^{\mathrm{T}} \mathbf{f}^{v}(\mathbf{v}, t) \mathrm{d} v
$$

In most of the cases, the vector of volume external forces can be written by means of a separation of variables, where $\mathbf{F}^{v}(\mathbf{v})$ depends on the spatial variable $\mathbf{v}$ and $\gamma(t)$ is a function of time, as follows

$$
\mathbf{f}^{v}(\mathbf{v}, t)=\mathbf{F}^{v}(\mathbf{v}) \gamma(t)
$$

Thus the generalised force can be written as

$$
\mathbf{Q}_{\mathbf{q}}(t)=\int_{\text {Volume }} \mathbf{\Phi}(\mathbf{v})^{\mathrm{T}} \mathbf{F}^{v}(\mathbf{v}) \mathrm{d} v \gamma(t)=\widetilde{\mathbf{F}} \gamma(t)
$$

Now the Equations from (19) to (22), (30), (33) and (37) are substituted into Equation (18), and it results

$$
\begin{aligned}
\ddot{\mathbf{q}}+\left(2 \Omega \widetilde{\mathbf{J}}+2 \Omega\left(\widetilde{\mathbf{G}}^{\mathrm{T}}+\widetilde{\mathbf{J}}^{\mathrm{T}}\right)\right) \dot{\mathbf{q}} \\
\quad+\left(\widetilde{\mathbf{K}}+\Omega^{2}(\widetilde{\mathbf{G}} \widetilde{\mathbf{G}}+\widetilde{\mathbf{G}} \widetilde{\mathbf{J}}-\widetilde{\mathbf{J}} \widetilde{\mathbf{G}}-\widetilde{\mathbf{J}} \widetilde{\mathbf{J}}-\widetilde{\mathbf{E}})\right) \mathbf{q}=\widetilde{\mathbf{F}} \gamma(t)+\Omega^{2} \widetilde{\mathbf{L}}
\end{aligned}
$$

Simplifying the last expression, it results

$$
\ddot{\mathbf{q}}-2 \Omega \widetilde{\mathbf{G}} \dot{\mathbf{q}}+\left(\widetilde{\mathbf{K}}+\Omega^{2} \widetilde{\mathbf{C}}\right) \mathbf{q}=\widetilde{\mathbf{F}} \gamma(t)+\Omega^{2} \widetilde{\mathbf{L}}
$$

With the matrix $\widetilde{\mathbf{C}}$ being defined as

$$
\widetilde{\mathbf{C}}=\widetilde{\mathbf{G}} \widetilde{\mathbf{G}}+\widetilde{\mathbf{G}} \widetilde{\mathbf{J}}-\widetilde{\mathbf{J}} \widetilde{\mathbf{G}}-\widetilde{\mathbf{J}} \widetilde{\mathbf{J}}-\widetilde{\mathbf{E}}
$$

It must be drawn attention to the fact that the matrices $\widetilde{\mathbf{G}}, \widetilde{\mathbf{J}}, \widetilde{\mathbf{K}}, \widetilde{\mathbf{E}}, \widetilde{\mathbf{F}}$ and $\widetilde{\mathbf{L}}$ are constant matrices and they are computed at the beginning of the simulation. Equation (39) is linear and it is expressed in a non-rotating reference frame.

\subsection{Contact model}

The simulation tool implements the model of Hertz for computing the distribution of normal pressure at the contact patch. The tangential contact problem is solved by 
adopting the FASTSIM algorithm. FASTSIM provides a good precision when calculating the relationship between the creepages and contact forces and, in addition, presents the advantage of a low computational cost since it is based on a simplified elastic theory. However, when using FASTSIM the accuracy of the calculation of the local slip at the contact patch may be affected by the adoption of a simplified theory.

\subsection{Wear model}

In the present work, the model of wear adopted for modelling the sliding wear at the wheel-rail contact is the Archard's model. According to this theory the wear rate $\dot{w}$ $[\mathrm{m} / \mathrm{s}]$ can be expressed as follows

$$
\dot{w}=\frac{k_{w}}{H} p_{z}\|\mathbf{s}\|
$$

where $p_{z}$ is the normal pressure applied to the surface, $\|\mathbf{s}\|$ is the modulus of the local slip, $k_{w}$ is the wear coefficient, which depends on the normal load and on the slip velocity, and $H$ is the material hardness. It is assumed that the wear coefficient is independent of the variations of the normal contact tractions or local slips at the contact patch. In the present study, the value of the relation $k_{w} / H$ is chosen to be $3.4 \cdot 10^{-14} \mathrm{~m}^{2} / \mathrm{N}$ [17].

For each calculation instant, the wear rate is obtained at the centre of each element of the discretization used by FASTSIM and it is transferred to a regular mesh of nodes on the running surface of the rail. Finally, the wear rates in each node of the rail are integrated over the time, resulting, thus, the wear depth at each rail node.

After each iteration loop of the simulation tool (see Figure 1), the railhead profile is modified according to the wear depth calculated in the last simulation.

\section{The influence of the wheelset angular velocity}

Table 3 shows the natural frequencies of the seven first modes of a free wheelset together with the multiplicity of the vibration modes. These natural frequencies correspond to those of the non-rotating wheelset. The effect of the angular velocity of the wheelset on the natural frequencies of the free wheelset can be analysed through the Frequency Response Function. The expression that can be derived from Equation (39) for computing the Frequency Response Function (FRF) is

$$
\mathbf{H}(\omega)=\boldsymbol{\Phi}^{\mathrm{T}}\left[-\omega^{2} \mathbf{I}-i 2 \omega \Omega \widetilde{\mathbf{G}}+\widetilde{\mathbf{K}}+\Omega^{2} \widetilde{\mathbf{C}}\right]^{-1} \boldsymbol{\Phi},
$$

in which $\mathbf{I}$ is the identity matrix and $i$ is the imaginary unit. The direct receptance $H_{z z}(\omega)$ represents the response of the contact point of the wheelset in the vertical direction when the excitation is applied to the same point in the same direction. In Figure 3, the direct receptance is plotted for two velocities of the vehicle: 0 and 100 $\mathrm{km} / \mathrm{h}$. This figure shows, when the velocity of the vehicle is zero, the resonance peaks corresponding to the frequency range from $0 \mathrm{~Hz}$ up to $400 \mathrm{~Hz}$ (see Table 3). When the angular velocity of the wheelset is non-zero, the resonance peaks related to the wheelset modes with multiplicity 2 (bending modes of the wheelset and wheel modes) split into 
two different peaks, which can be associated with the backward and forward modes [6]. However, the peaks related to the modes with multiplicity 1 remain unchanged.

Figure 4 shows the cross receptance $H_{x z}(\omega)$, that is, the response of the contact point of the wheelset in the longitudinal direction ( $x$-axis coincident with the track axis direction) when the excitation is in the vertical direction. Considering the symmetry of revolution of the wheelset, the level of this response should be zero. However, at the sight of Figure 4, this fact does not happen when the angular velocity of the wheelset is different from zero. Again, it can be appreciated in Figure 4 that when the velocity of the wheelset increases, the two resonance peaks derived from the modes with multiplicity 2 split into two different peaks. The peaks 2, 4, 7 and 9 correspond to the backward modes, while the peaks 3, 5, 8 and 10 are associated with the forward modes. The peaks 1 and 6 are related to the first torsional mode and the first umbrella mode, which are of multiplicity 1.

In the Campbell diagram (Figure 5), the frequencies of the vibration modes of the mounted wheelset are represented in function of the velocity of the vehicle.

The modes with multiplicity 1 are, when ordered according to growing frequencies, the first torsional mode, and the symmetric and the anti-symmetric umbrella modes. The modes with multiplicity 2 (the three first bending modes of the wheelset and the wheel modes) split into two different modes, the backward and forward modes, when the vehicle velocity is different from zero. For the forward modes, the frequency increases as the velocity rises, while for the backward modes, the frequency decreases with the velocity. Thus, the difference between the frequencies of the backward and the forward modes increases as the vehicle speed rises.

Particularly interesting for this research work is the coincidence of two vibration modes of the wheelset. It can be observed in Figure 5 that there are two intersection points of the line of the backward wheel mode with the lines of the forward and backward third bending modes of the wheelset. The left-hand intersection point corresponds to the coincidence of the backward wheel mode with the forward third bending mode of the wheelset (B-F from now on). It occurs at a frequency of $360.8 \mathrm{~Hz}$ and a vehicle velocity of $142 \mathrm{~km} / \mathrm{h}$. The right-hand intersection point is due to the coincidence of the backward wheel mode with the backward third bending mode of the wheelset (B-B from now on), and it corresponds to a frequency of $352.5 \mathrm{~Hz}$ and a vehicle velocity of $198 \mathrm{~km} / \mathrm{h}$.

Figures 6 and 7 show the direct and cross receptances, respectively, in the frequency range from approximately $342 \mathrm{~Hz}$ up to $375 \mathrm{~Hz}$ for the above mentioned vehicle velocities. As can be observed in both figures, the resonance peak corresponding to the forward third bending mode of the wheelset occurs at a frequency of $360.8 \mathrm{~Hz}$ when the vehicle velocity is $142 \mathrm{~km} / \mathrm{h}$ and the resonance peak of the backward third bending mode of the wheelset appears at a frequency of $352.5 \mathrm{~Hz}$ when the vehicle velocity is $198 \mathrm{~km} / \mathrm{h}$. It cannot be inferred from the analysis of these wheelset receptances that the resulting wear depth will be higher at a certain resonance frequency than that at the others, that is, the wheelset receptances do not provide information about whether or not rail corrugation grows at certain resonance frequencies. 
Table 3. Vibration modes of the wheelset

\begin{tabular}{|c|c|c|c|}
\hline Modes of the flexible wheelset & Multiplicity & Frequency $(\mathrm{Hz})$ & Mode shape \\
\hline $\begin{array}{l}\text { First torsional } \\
\text { mode }\end{array}$ & 1 & 81.5 & \\
\hline $\begin{array}{l}\text { First horizontal } \\
\text { and vertical } \\
\text { bending modes }\end{array}$ & 2 & 102 & \\
\hline $\begin{array}{l}\text { Second horizontal } \\
\text { and vertical } \\
\text { bending modes }\end{array}$ & 2 & 179 & \\
\hline $\begin{array}{l}\text { First umbrella } \\
\text { mode }\end{array}$ & 1 & 298 & \\
\hline $\begin{array}{l}\text { Third horizontal } \\
\text { and vertical } \\
\text { bending modes }\end{array}$ & 2 & 357 & \\
\hline $\begin{array}{l}\text { Modes of the } \\
\text { wheel with two } \\
\text { nodal diameters } \\
\text { and no nodal } \\
\text { circle }\end{array}$ & 4 & 383 & \\
\hline $\begin{array}{l}\text { Second umbrella } \\
\text { mode }\end{array}$ & 1 & 436 & \\
\hline
\end{tabular}




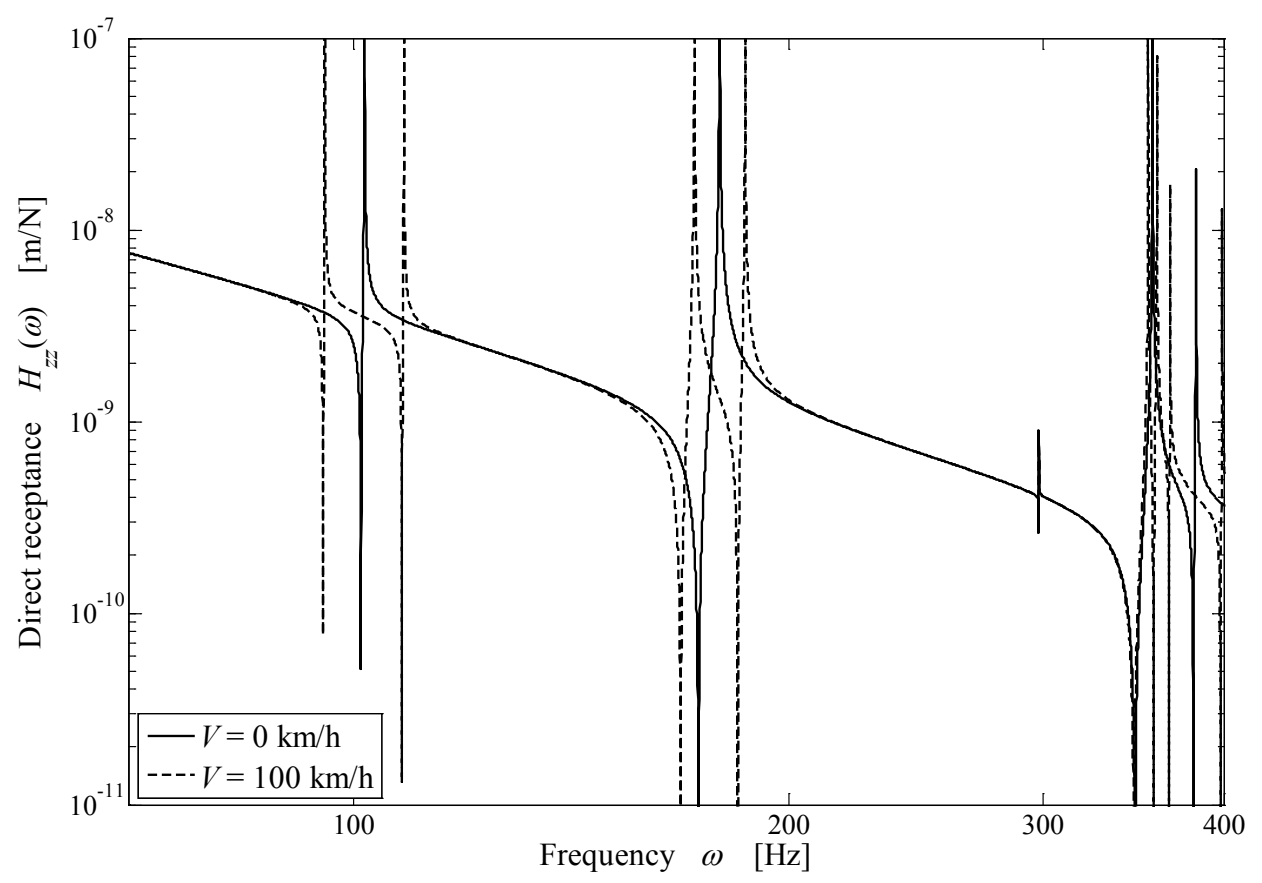

Figure 3. Direct receptance modulus $H_{z z}$ in function of the frequency when the velocity of the vehicle $V$ is $0 \mathrm{~km} / \mathrm{h}$ and $100 \mathrm{~km} / \mathrm{h}$. The excitation is applied to the wheel at the contact point in the vertical direction and the measure of the response is taken at the same point and in the same direction.

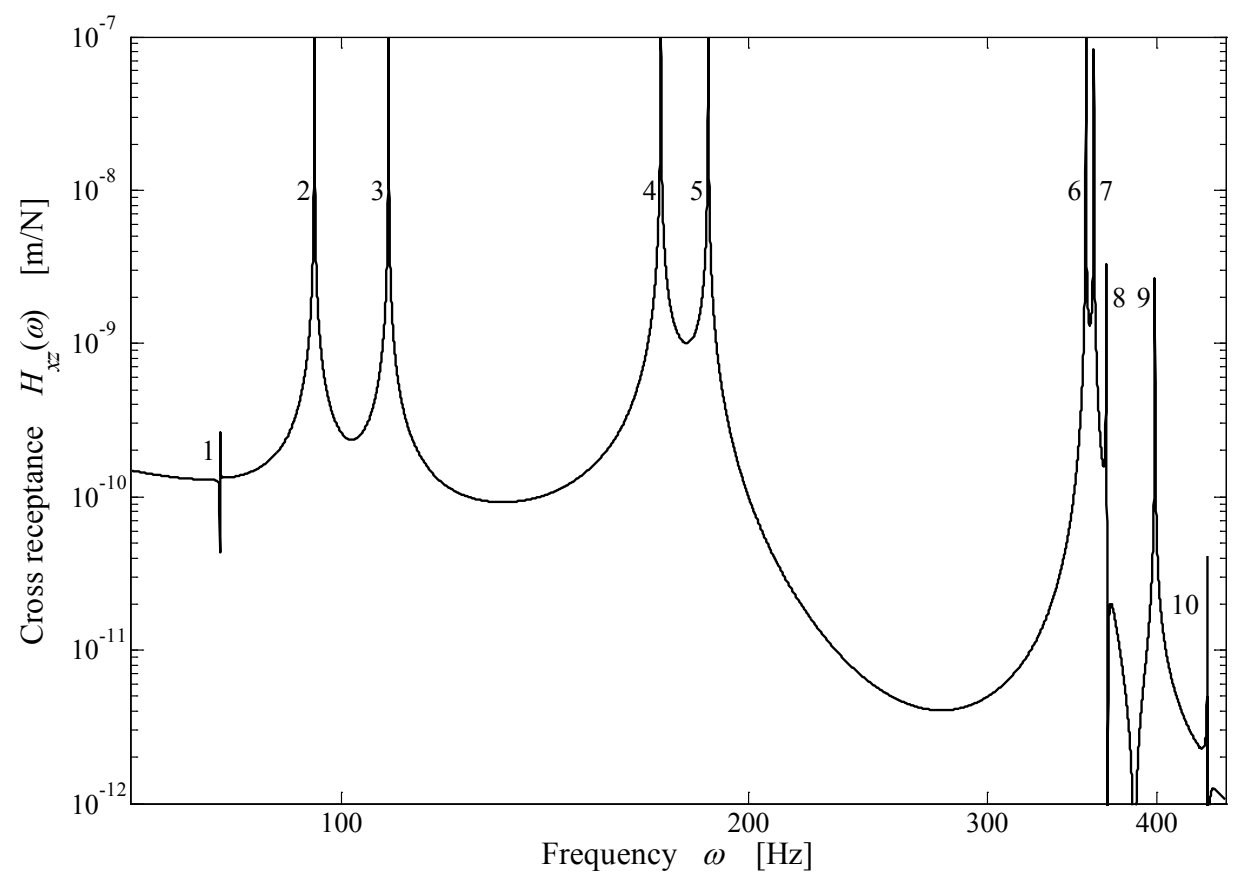

Figure 4. Cross receptance modulus $H_{x z}$ in function of the frequency when the velocity of the vehicle is $100 \mathrm{~km} / \mathrm{h}$. The excitation is applied to the wheel at the contact point in the vertical direction and the measure of the response is taken at the same point in the longitudinal direction. Peak 1: 1st torsional mode; Peaks $2 \&$ 3: 1 st bending mode (backward and forward, respectively); Peaks 4 \& 5: 2nd bending mode (backward, forward); Peak 6:1st umbrella mode; Peaks $7 \&$ 8: 3rd bending mode (backward, forward); Peaks 9 \& 10: wheel modes (backward, forward). 


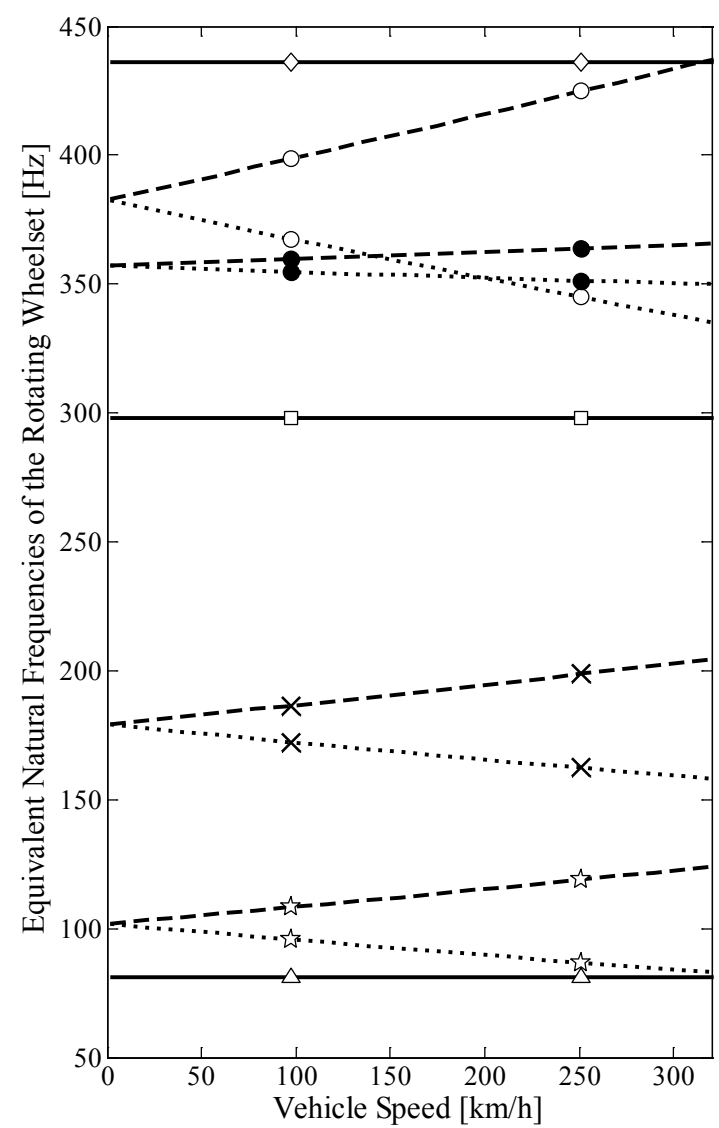

Figure 5. Campbell diagram for the railway wheelset. Modes with multiplicity 1 (in continuous trace), forward modes (in dashed trace), backward modes (in dotted trace). First torsional mode (triangle), first bending mode (five-pointed star), second bending mode (cross), first umbrella mode (square), third bending mode (black circle), wheel modes (white circle) and second umbrella mode (diamond).

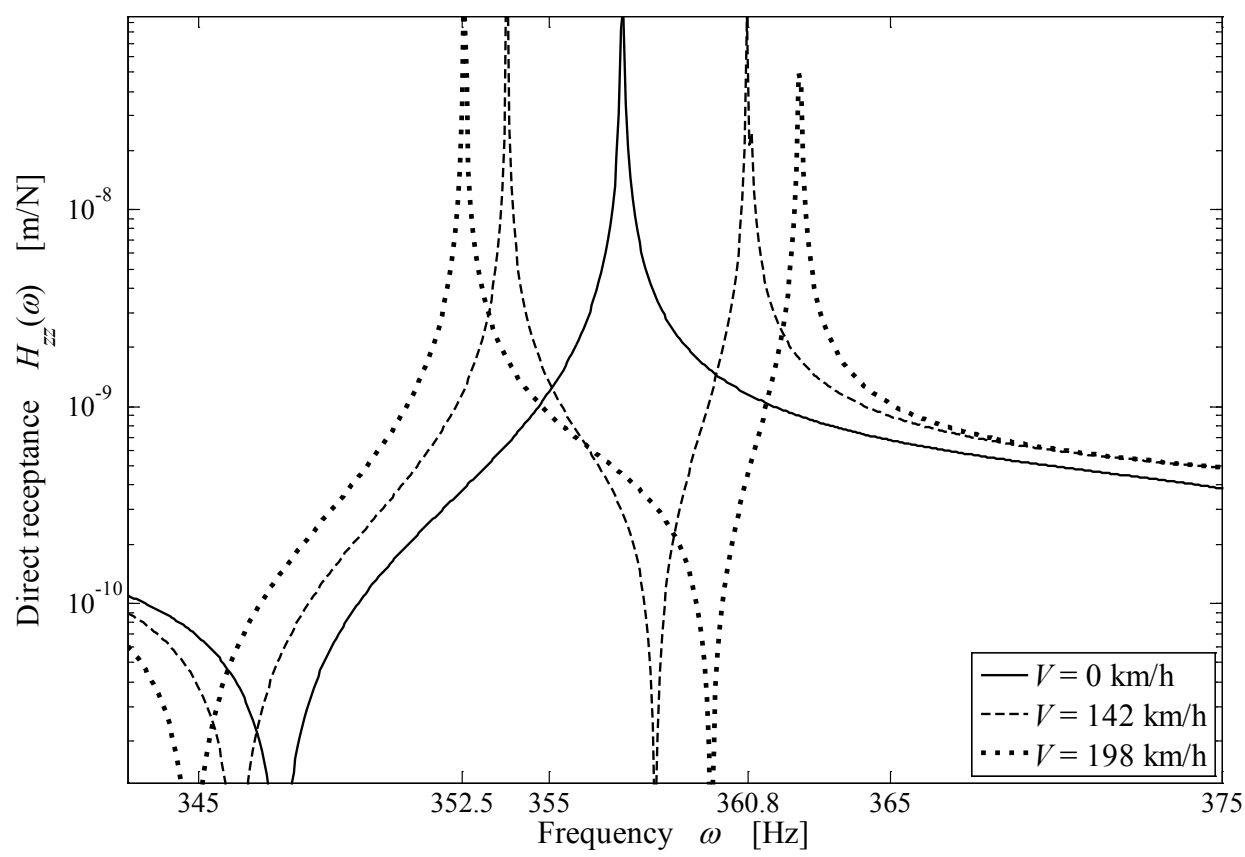

Figure 6. Direct receptance modulus $H_{z z}$ in function of the frequency when the velocity of the vehicle $V$ is $0 \mathrm{~km} / \mathrm{h}, 142 \mathrm{~km} / \mathrm{h}$ and $198 \mathrm{~km} / \mathrm{h}$. The excitation is applied to the wheel at the contact point in the vertical direction and the measure of the response is taken at the same point and in the same direction. 


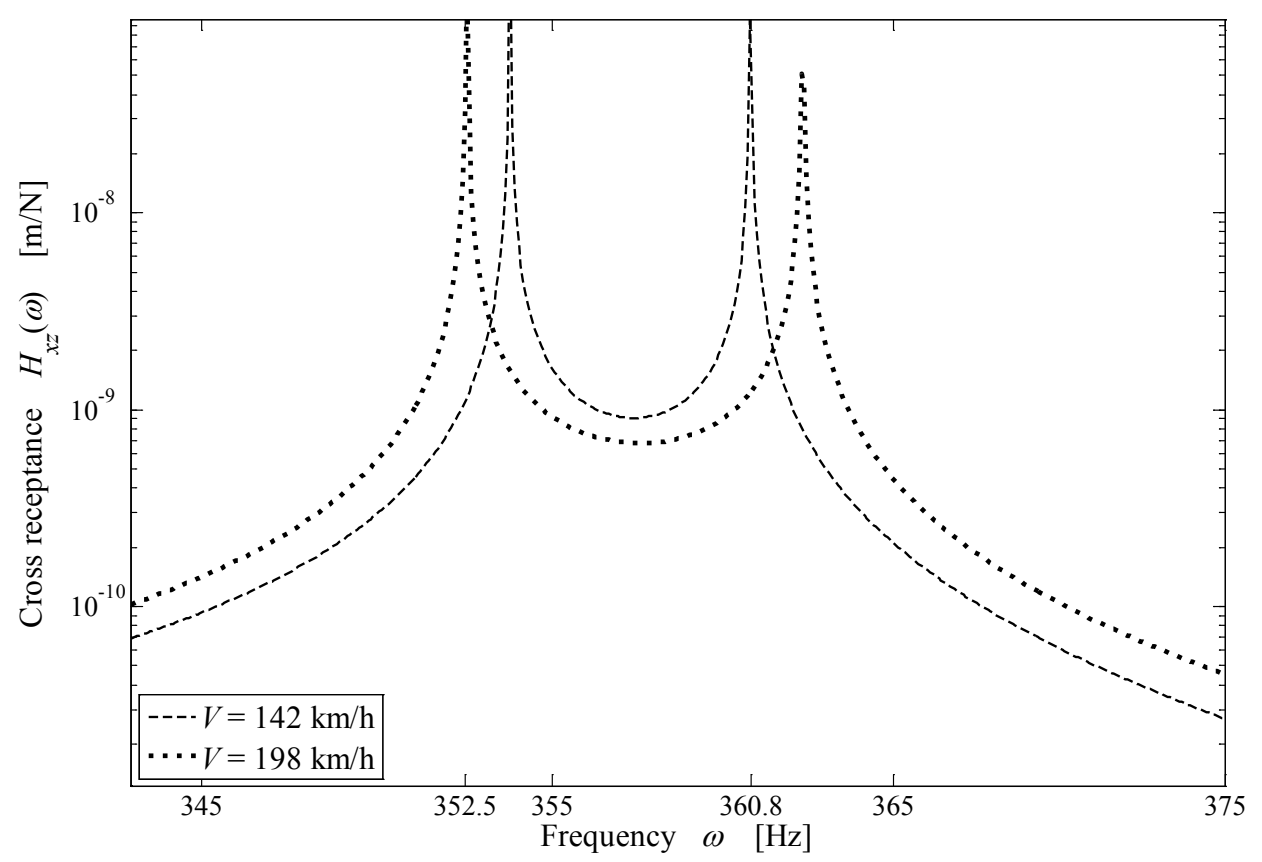

Figure 7. Cross receptance modulus $H_{x z}$ in function of the frequency when the velocity of the vehicle is $142 \mathrm{~km} / \mathrm{h}$ and $198 \mathrm{~km} / \mathrm{h}$. The excitation is applied to the wheel at the contact point in the vertical direction and the measure of the response is taken at the same point in the longitudinal direction.

\section{Results from the wear simulation}

In a previous work [10], it was concluded that the vibratory responses of the different models of the mounted wheelset (the rigid wheelset model, the non-rotating and the rotating flexible wheelset models) are virtually coincident, except when the vibration modes of the flexible wheelset models are excited. A more recent study [17] analysed the wear computed through three different modes at arbitrary velocities and it showed similar results and very small wear. Therefore, it was decided to study the rotating flexible wheelset model and to compare the results with those from the rigid wheelset model when the wavelength of the rail roughness excites the vibration modes of the wheelset determined by using the Campbell diagram.

Certain combinations of angular velocity of the wheelset and excitation frequency can lead to the excitation of several vibration modes with the consequent generation of complex dynamics. These cases occur for the configurations associated with the points in which two lines in the Campbell diagram intersect. In this section, the effect of the excitation of the B-F modes and the B-B modes on the evolution of rail corrugation is analysed.

To this aim, an initial sinusoidal roughness on the running surface of the rails is assumed. For the first simulation, the wavelength of the roughness is set to be $11 \mathrm{~cm}$, the amplitude, $100 \mu \mathrm{m}$ and the vehicle velocity, $142 \mathrm{~km} / \mathrm{h}$. Thus, the excitation frequency is $360.8 \mathrm{~Hz}$, at which both the backward wheel mode and the forward third bending mode of the wheelset are excited (B-F modes). In the second simulation, the wavelength of the roughness is assumed to be $15.6 \mathrm{~cm}$, the amplitude, $100 \mu \mathrm{m}$ and the vehicle velocity, $198 \mathrm{~km} / \mathrm{h}$, with the objective that the excitation frequency be $352.5 \mathrm{~Hz}$. 
At this frequency, the backward wheel mode and the backward third bending mode of the wheelset are excited (B-B modes).

Figure 8 shows the contact forces, namely longitudinal, lateral and vertical contact forces, resulting from the first simulation. It must be highlighted that no force related to a motor torque was implemented. Therefore the resulting longitudinal and transversal forces are consequences of the torsion vibrations, the rotation of the section of the rail and the proper dynamics of the wheelset. It can be observed that the use of the rotating flexible wheelset model leads to higher contact forces than when using the rigid wheelset model, especially in the cases of the longitudinal and lateral contact forces. In Figure 9, the contact forces from the second simulation are plotted. Again, the contact forces calculated through the rotating flexible wheelset model are higher than those obtained through the rigid model, except for the vertical force.

The animations of the wheelset dynamics for the above mentioned velocities can be found in the electronic annexes of this article.

Figures 10 and 11 show the wear depth on the running surface of the rails after a wheelset passage when the vehicle velocities are $142 \mathrm{~km} / \mathrm{h}$ and $198 \mathrm{~km} / \mathrm{h}$, respectively. In these figures, the wear depth is plotted together with the initial roughness (without scale) for two sleeper bays. It can be observed that the wear depth resulting when the B$\mathrm{F}$ modes are excited (Figure 10) is approximately 5 times higher than that produced when the B-B modes are excited (Figure 11). In both simulations, the wear occurs at the crests and the troughs with a frequency double that of the initial roughness.

Figures 12 and 13 show the evolution of the railhead profile after 100000 wheelset passages. The worn rail profile after 100000 wheelset passages is obtained through 100 simulations. After each simulation the rail profile is updated with the wear depth multiplied by a factor of 1000 since it was observed that 10 simulations in which the wear was multiplied by 100 passages led to the same worn rail profile as one simulation in which the wear depth was multiplied by 1000 . While the effect of the excitation of the B-B modes on the change of the railhead profile seems to be almost negligible at the sight of Figure 13, it appears that the excitation of the B-F modes has a relevant role in the evolution of rail corrugation (see Figure 12). In Figure 12, it can be noted that the change in the railhead profile is localised on the crest and the trough of the initial profile, with wear being deeper at the troughs. 
(a)

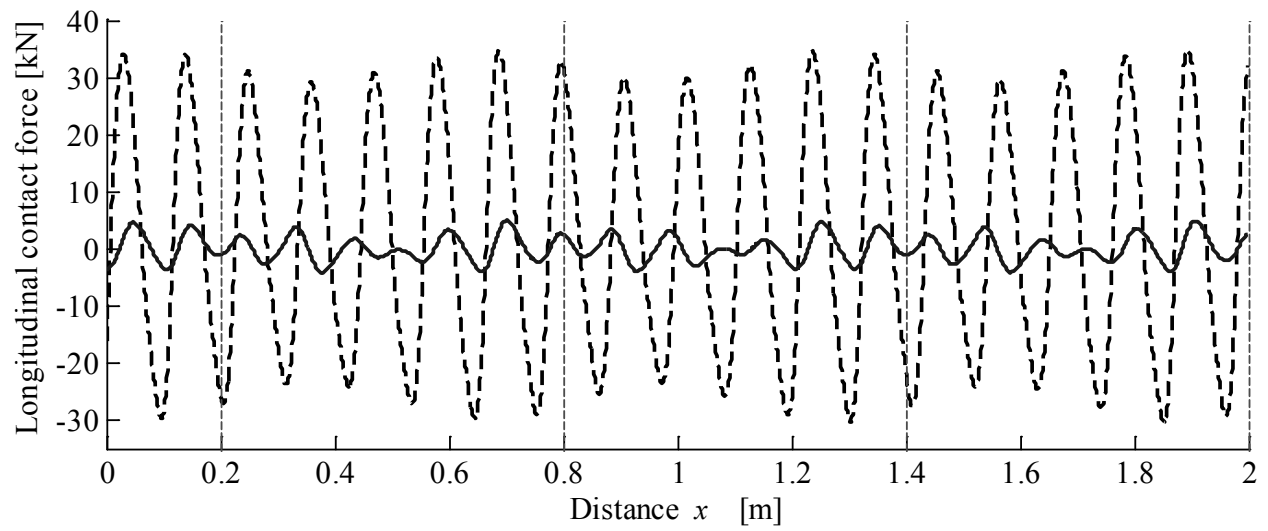

(b)

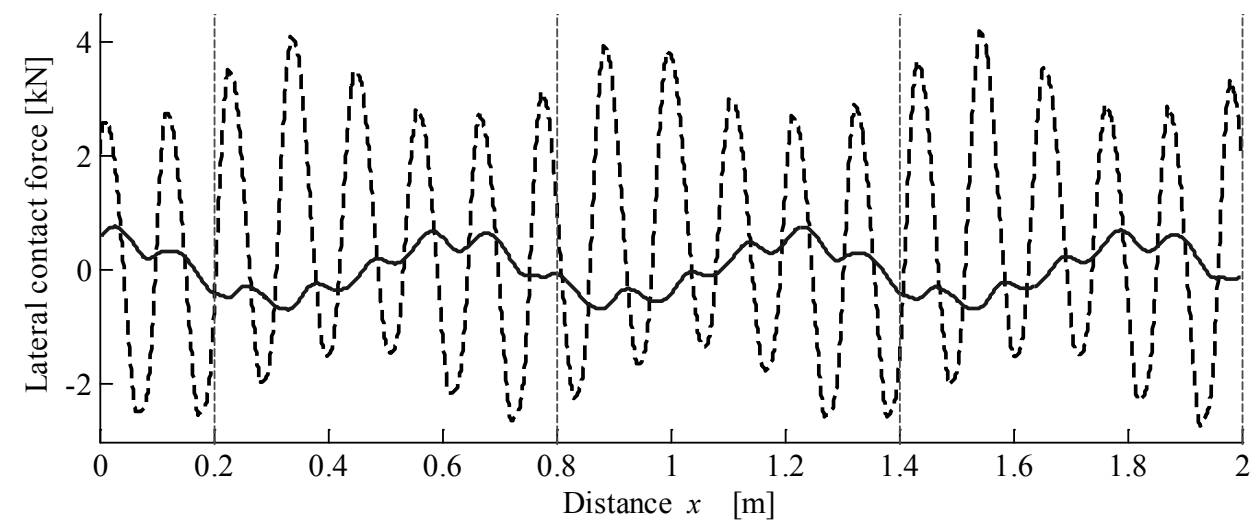

(c)

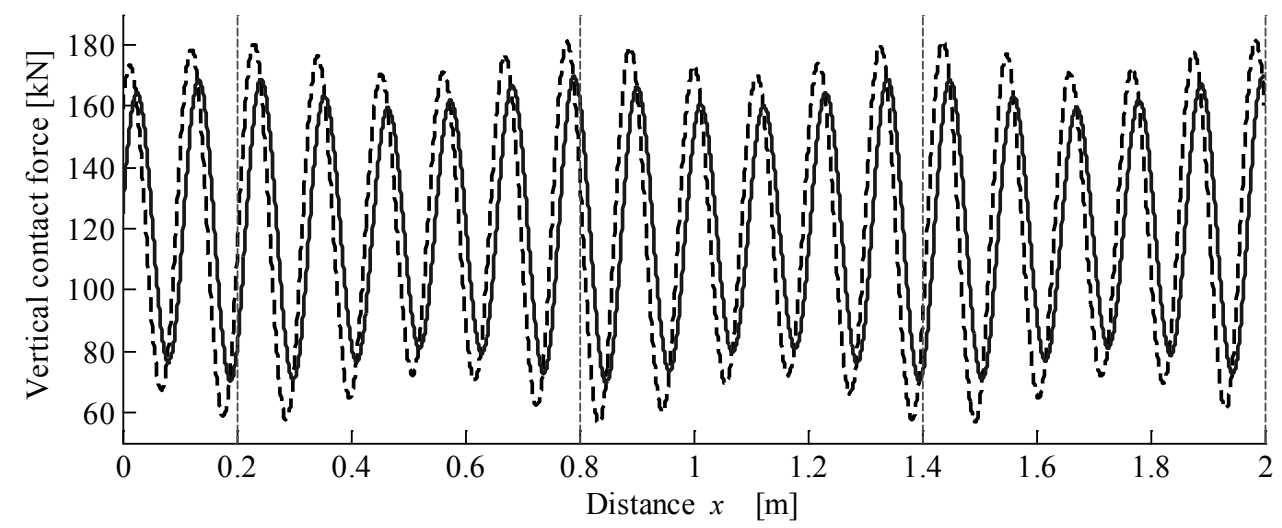

Figure 8. Contact forces computed through the rotating flexible wheelset model (dashed line), and the rigid wheelset model (continuous line) for a vehicle velocity of $142 \mathrm{~km} / \mathrm{h}$ : (a) longitudinal force, (b) lateral force, (c) vertical force. (The vertical dashed lines indicate the position of the sleepers). 
(a)

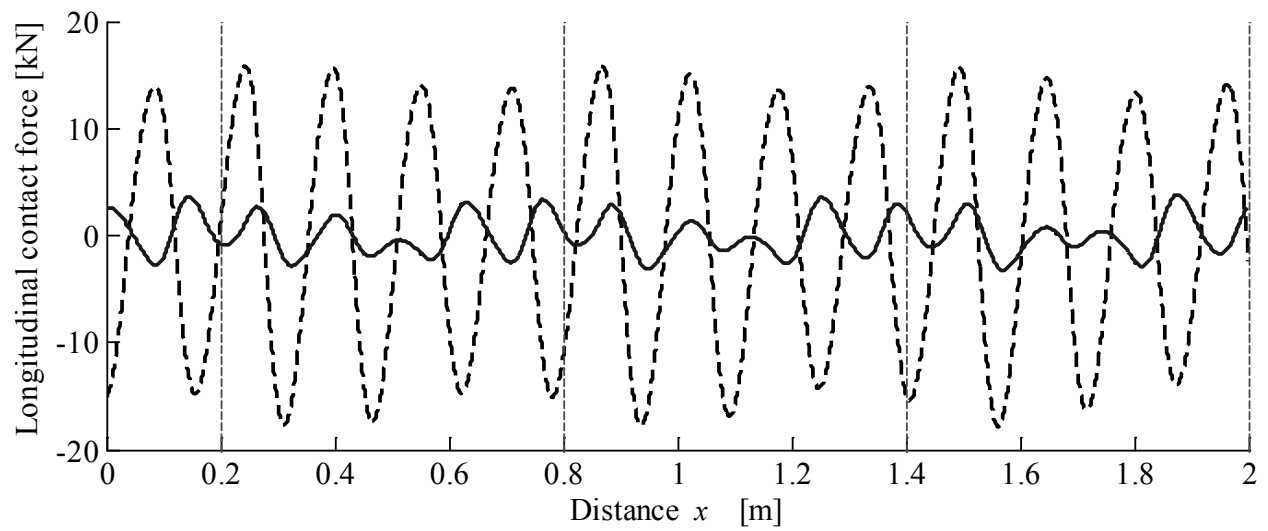

(b)

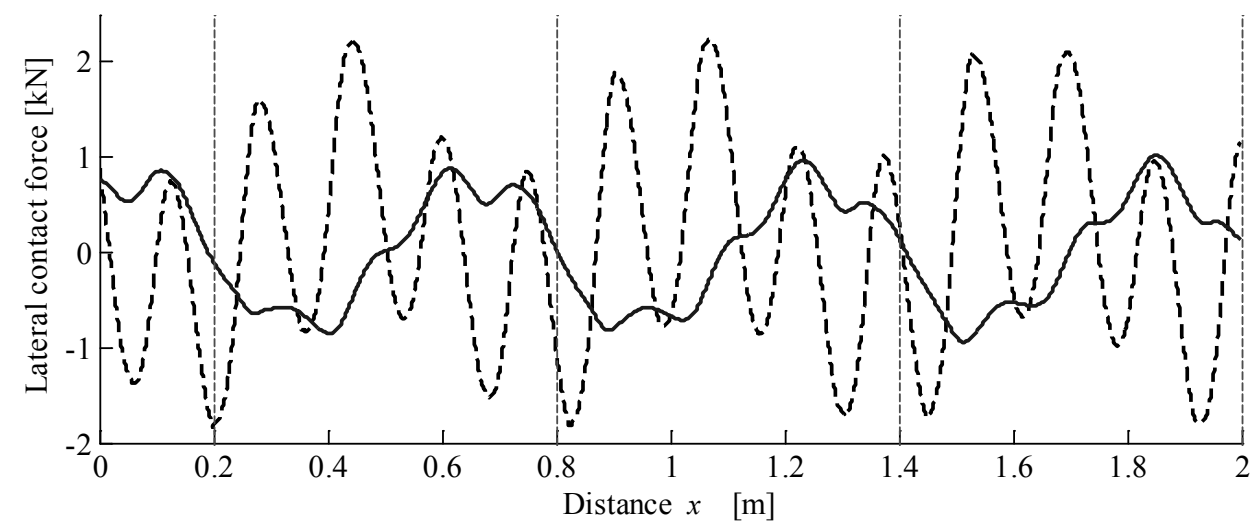

(c)

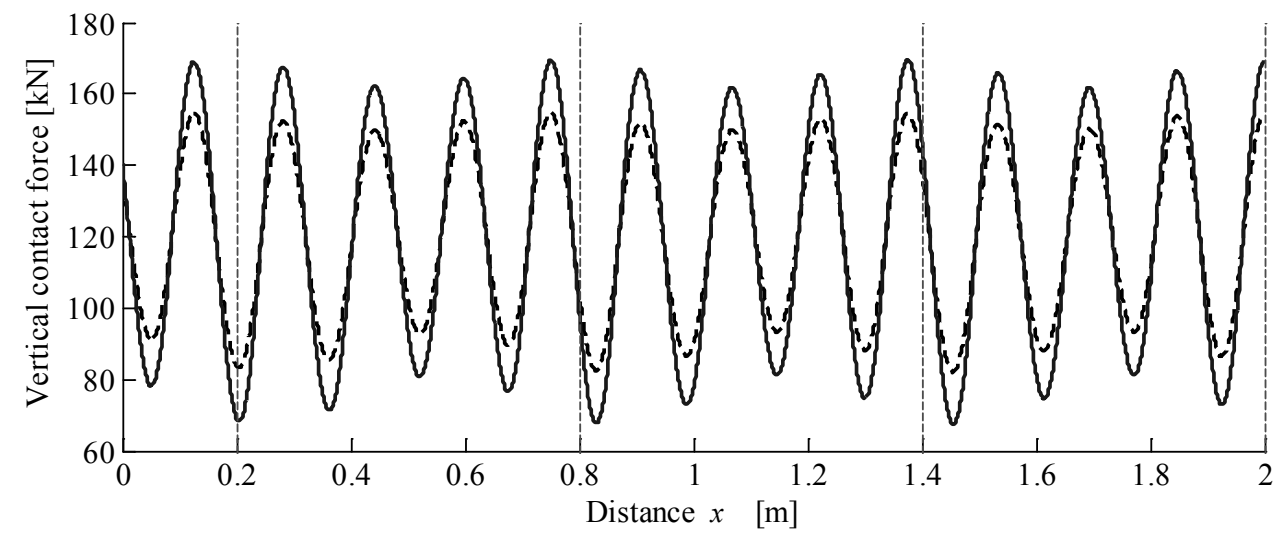

Figure 9. Contact forces computed through the rotating flexible wheelset model (dashed line), and the rigid wheelset model (continuous line) for a vehicle velocity of $198 \mathrm{~km} / \mathrm{h}$ : (a) longitudinal force, (b) lateral force, (c) vertical force. (The vertical dashed lines indicate the position of the sleepers). 


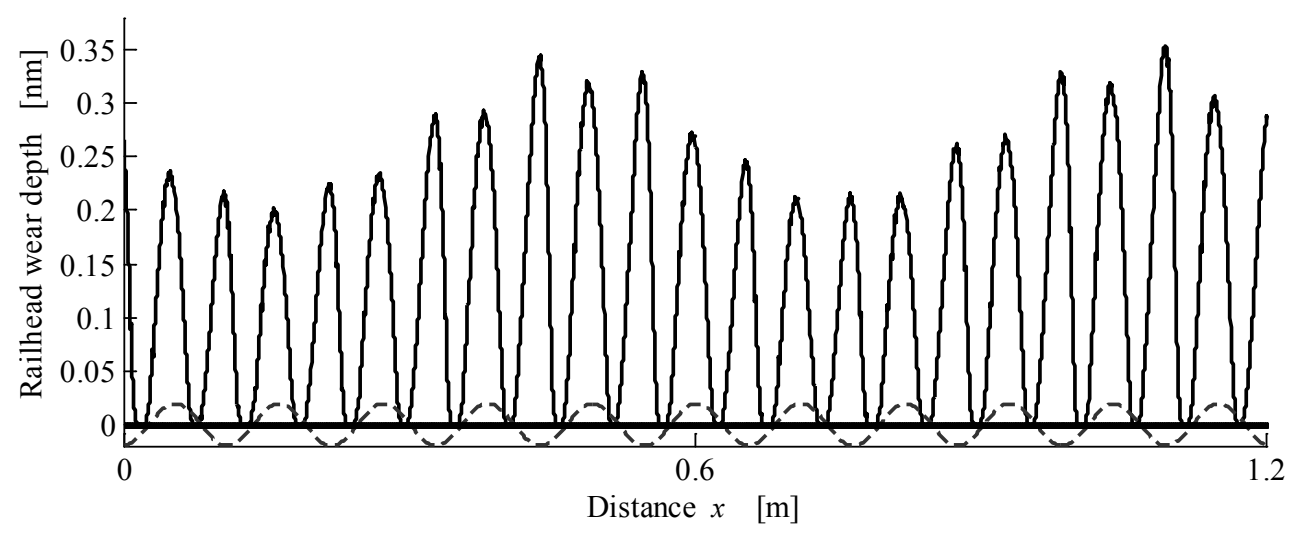

Figure 10. Depth of the railhead wear for a wheelset passage at the vehicle velocity of $142 \mathrm{~km} / \mathrm{h}$ (continuous line), initial rail roughness (dashed line, without scale)

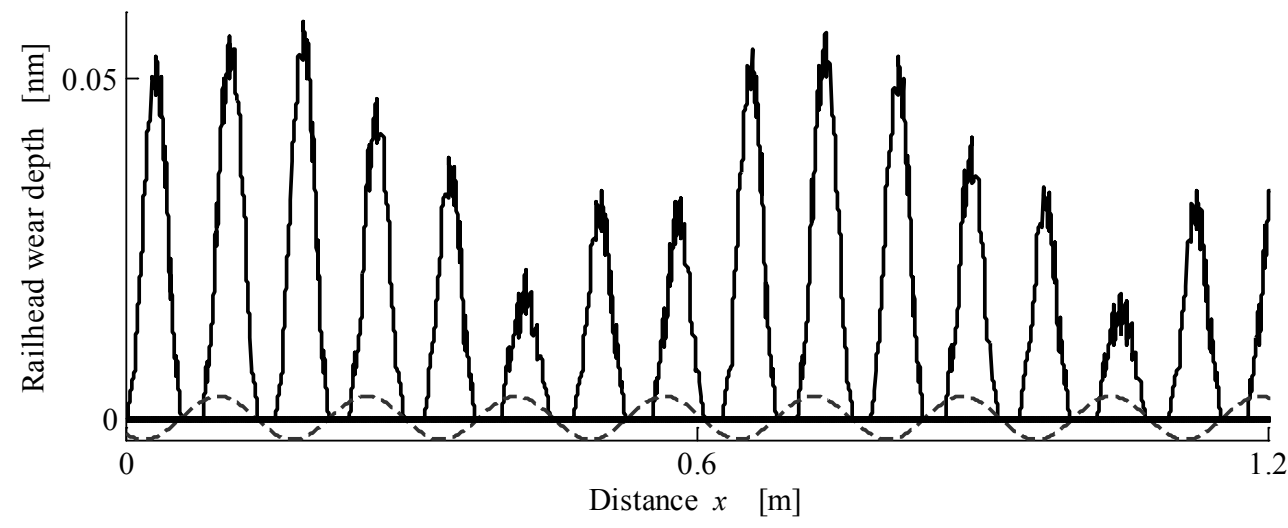

Figure 11. Depth of the railhead wear for a wheelset passage at the vehicle velocity of $198 \mathrm{~km} / \mathrm{h}$ (continuous line), initial rail roughness (dashed line, without scale). 


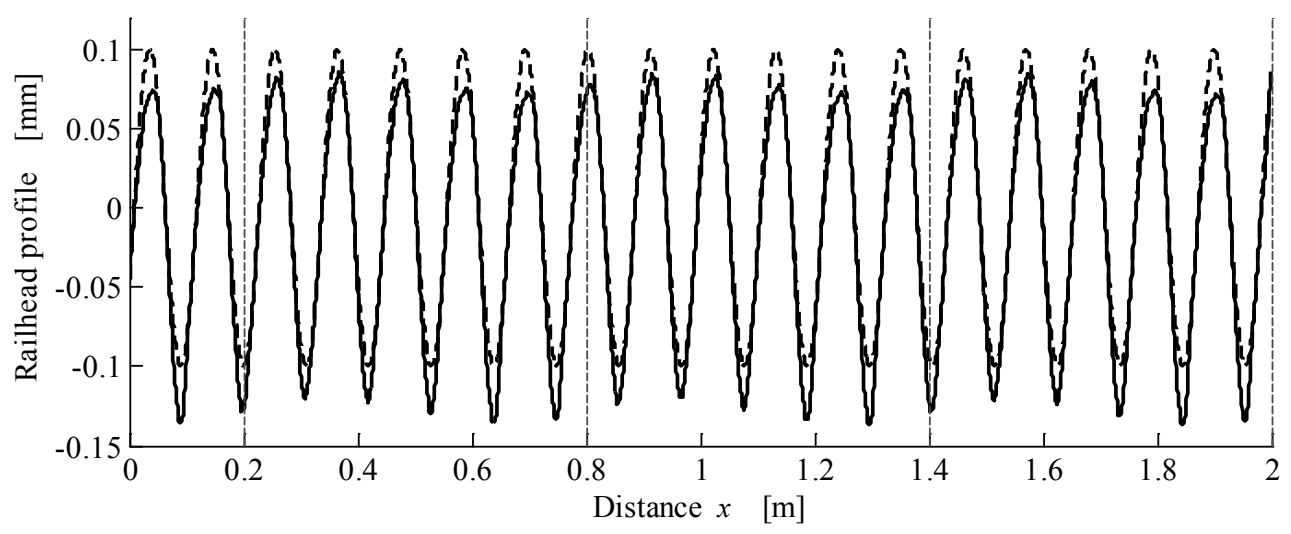

Figure 12. Railhead profile after 100000 wheel passages for a vehicle velocity of $142 \mathrm{~km} / \mathrm{h}$ (continuous line) and initial profile (dashed line). (The vertical dashed lines indicate the position of the sleepers).

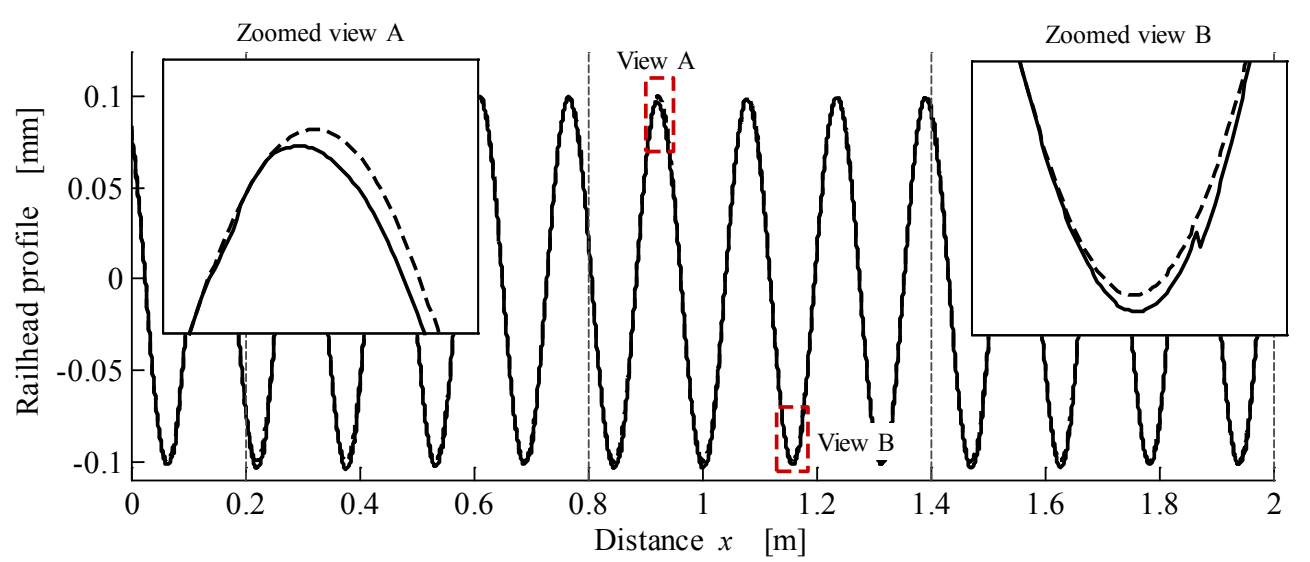

Figure 13. Railhead profile after 100000 wheel passages for a vehicle velocity of $198 \mathrm{~km} / \mathrm{h}$ (continuous line) and initial profile (dashed line). (The vertical dashed lines indicate the position of the sleepers).

\section{Conclusions}

In this work, a simulation tool intended to study the evolution of the rail roughness has been presented. The simulation tool consists of a cyclic vehicle-track system model, a rotating flexible wheelset model, a wheel-rail contact model and a wear theory.

The model of the vehicle-track system assumes the hypotheses of periodicity, and consequently it is considered as cyclic. The adoption of hypotheses of periodicity permit to eliminate the border effects and to perform integrations without time limitation since the vehicle does not reach the border of the track, as happens in finite track models.

Regarding the wheelset model, it has been taken into account its flexibility and the effects associated with the rotation, namely the gyroscopic effect and the effect of mobile force of the contact point with respect to the wheelset. The formulation of the flexible wheelset model uses a set of Eulerian modal coordinates and it is based on the position of a spatial point in opposition to the Lagragian formulation commonly used in mechanical engineering. The Eulerian formulation presents a series of advantages. Firstly, the wheel-rail contact force is applied on the wheelset at spatial points, whose positions remain unchanged. Consequently, the term of the generalised force becomes 
significantly simplified. Secondly, from another viewpoint, the wheel-rail contact theories were developed through Eulerian coordinates, and therefore the formulation of the creep velocities is notably simplified, since the use of Lagrangian coordinates forces to implement convective terms complicates significantly the calculation. Finally, the Eulerian formulation enables the generation of coherent solid-air meshes in acoustic problems since the rotation of the mesh is not required.

In this research, further insight into the mechanisms related to the dynamics of the wheel-track system that promote the growth of rail corrugation has been gained. By using the Campbell diagram, it has been identified the vibration modes of the wheelset responsible for a complex dynamics able to produce high forces at the wheel-rail contact and wear. Specifically, it has been analysed the coupled influence of the third bending mode of the wheelset and the wheel modes on the growth of rail corrugation. The possible wavelength-fixing mechanism that has been identified is not constant in frequency, but it depends on the velocity of the vehicle.

From the results obtained through the simulations, it can be concluded that the dynamics of the rotating flexible wheelset, specifically the B-F mode, might give rise to generation mechanisms of rail corrugation. However, this theoretical study is not definitive since further numerical simulations and comparisons to experimental data are needed to confirm the influence of certain resonances of the rotating flexible wheelset on the development of rail corrugation.

\section{Acknowledgements}

The authors gratefully acknowledge the support for this work provided by the Project TRA2010-15669 (Ministerio de Ciencia e Innovación) and the valuable help of P. Torstersson from Chalmers University, who suggested the use of the Campbell diagram in this investigation.

\section{References}

[1] K. H. Oostermeijer, Review on short pitch rail corrugation studies, Wear 265 (2008) 1231-1237.

[2] S. L. Grassie, J. W. Edwards, Development of corrugation as a result of varying normal load, Wear 265 (2008) 1150-1155.

[3] S. L. Grassie, J. Kalousek, Rail corrugation: characteristics, causes and treatments, Proc. IMechE, Part F: J. Rail and Rapid Transit 207 (1993) 57-68.

[4] S. L. Grassie, Rail corrugation: characteristics, causes, and treatments. Proc. IMechE, Part F: J. Rail and Rapid Transit 223 (2009) 581-596.

[5] G. Genta, Dynamics of rotating systems, Springer, New York 0-387-20936-0, 2005.

[6] J. Fayos, A. Rovira, L. Baeza, J. Carballeira, Rotating shaft analytical response in adimensional form, Proceedings of the Thirteenth International Congress on Sound and Vibration, Vienna, 2006.

[7] I. Gómez and E. G. Vadillo, A linear model to explain short pitch corrugation on rails, Wear 255 (2003) 1127-1142.

[8] D. J. Thompson, Wheel-rail noise generation, part V: inclusion of wheel rotation, Journal of Sound and Vibration 161 (1993) 467-482.

[9] J. Fayos, L. Baeza, F. D. Denia, J. E. Tarancón, An Eulerian coordinate-based method for analysing the structural vibrations of a solid of revolution rotating about its main axis, Journal of Sound and Vibration 306 (2007) 618-635. 
[10] L. Baeza, J. Fayos, A. Roda, R. Insa, High frequency railway vehicle-track dynamics through flexible rotating wheelsets, Vehicle System Dynamics 46 (2008) 647-659.

[11] L. Baeza, H. Ouyang, A railway track dynamics model based on modal substructuring and a cyclic boundary condition, Journal of Sound and Vibration 330 (2011) 75-86.

[12] A.V. Vostroukhov, A.V. Metrikine, Periodically supported beam on a viscoelastic layer as a model for dynamic analysis of a high-speed railway track, International Journal of Solids and Structures 40 (2003) 5723-5752.

[13] D. J. Mead, Free wave propagation in periodically supported infinite beams, Journal of Sound and Vibration 11 (1970) 181-197.

[14] A. A. Shabana. Dynamics of multibody systems. Cambridge University Press, 1998.

[15] M. A. Brown, A. A. Shabana, Application of multibody methodology to rotating shaft problems, Journal of Sound and Vibration 204 (1997) 439-457.

[16] L. Baeza, P. Vila, A. Roda, J. Fayos, Prediction of corrugation in rails using a non-stationary wheel-rail contact model, Wear 265 (2008) 1156-1162.

[17] G. Xie, S.D. Iwnicki, P. Vila, L. Baeza, An investigation of rail corrugation with a coupled, flexible, rotating wheelset, a flexible track and a non-steady contact model, Proceedings of the Eighth International Conference on Contact Mechanics and Wear of Rail/Wheel Systems, Firenze, 2009.

\section{Appendix A}

Any scalar field $\gamma$ integrable on the domain of the solid of revolution has the following property

$$
\int_{\text {Volume }} \gamma(\mathbf{v}) \mathrm{d} v=\int_{\text {Volume }} \gamma(\overline{\mathbf{u}}) \mathrm{d} v .
$$

This is an evident result since the Jacobian determinant of the transformation (13) is 1.

\section{Appendix B}

Let us consider the matrix $\tilde{\mathbf{M}}$, which is defined as follows

$$
\tilde{\mathbf{M}}=\int_{\text {Volume }} \boldsymbol{\Phi}(\overline{\mathbf{u}})^{\mathrm{T}} \mathbf{M} \boldsymbol{\Phi}(\overline{\mathbf{u}}) \mathrm{d} v
$$

where $\mathbf{M}$ is a constant $3 \times 3$ matrix. Matrix $\mathbf{M}$ verifies that

$$
\mathbf{M}=\mathbf{A}^{\mathrm{T}} \mathbf{M} \mathbf{A}
$$

The transformation matrix $\mathbf{B}(t)$ does not affect if it is applied on the matrix $\tilde{\mathbf{M}}$, that is

$$
\mathbf{B}(t)^{\mathrm{T}} \tilde{\mathbf{M}} \mathbf{B}(t)=\tilde{\mathbf{M}}
$$

The proof is the following 


$$
\begin{aligned}
\mathbf{B}(t)^{\mathrm{T}} \tilde{\mathbf{M}} \mathbf{B}(t) & =\mathbf{B}(t)^{\mathrm{T}}\left(\int_{\text {Volume }} \boldsymbol{\Phi}(\overline{\mathbf{u}})^{\mathrm{T}} \mathbf{M} \boldsymbol{\Phi}(\overline{\mathbf{u}}) \mathrm{d} v\right) \mathbf{B}(t) \\
& =\int_{\text {Volume }} \mathbf{B}(t)^{\mathrm{T}} \boldsymbol{\Phi}(\overline{\mathbf{u}})^{\mathrm{T}} \mathbf{M} \boldsymbol{\Phi}(\overline{\mathbf{u}}) \mathbf{B}(t) \mathrm{d} v \\
& =\int_{\text {Volume }} \mathbf{B}(t)^{\mathrm{T}} \boldsymbol{\Phi}(\mathbf{v})^{\mathrm{T}} \mathbf{M} \boldsymbol{\Phi}(\mathbf{v}) \mathbf{B}(t) \mathrm{d} v \\
& =\int_{\text {Volume }} \boldsymbol{\Phi}(\overline{\mathbf{u}})^{\mathrm{T}} \mathbf{A}^{\mathrm{T}} \mathbf{M} \mathbf{A} \boldsymbol{\Phi}(\overline{\mathbf{u}}) \mathrm{d} v \\
& =\int_{\text {Volume }} \boldsymbol{\Phi}(\overline{\mathbf{u}})^{\mathrm{T}} \mathbf{M} \boldsymbol{\Phi}(\overline{\mathbf{u}}) \mathrm{d} v=\tilde{\mathbf{M}}
\end{aligned}
$$

\section{Appendix C}

The transformation matrix $\mathbf{B}(t)$ can be proved to be orthogonal. If Equation (8) is substituted into Equation (6), one obtains

$$
\boldsymbol{\Phi}(\mathbf{v}) \mathbf{B}(t)=\mathbf{A} \boldsymbol{\Phi}\left(\mathbf{A}^{\mathrm{T}} \mathbf{v}\right)
$$

Each side of the last equation are multiplied by itself (transposed) and the density, giving

$$
\rho \mathbf{B}(t)^{\mathrm{T}} \boldsymbol{\Phi}(\mathbf{v})^{\mathrm{T}} \boldsymbol{\Phi}(\mathbf{v}) \mathbf{B}(t)=\rho \boldsymbol{\Phi}\left(\mathbf{A}^{\mathrm{T}} \mathbf{v}\right)^{\mathrm{T}} \mathbf{A}^{\mathrm{T}} \mathbf{A} \boldsymbol{\Phi}\left(\mathbf{A}^{\mathrm{T}} \mathbf{v}\right)
$$

The Equation (C.2) is now integrated over the solid volume

$$
\mathbf{B}(t)^{\mathrm{T}}\left(\int_{\text {Volume }} \rho \boldsymbol{\Phi}(\mathbf{v})^{\mathrm{T}} \boldsymbol{\Phi}(\mathbf{v}) \mathrm{d} v\right) \mathbf{B}(t)=\int_{\text {Volume }} \rho \boldsymbol{\Phi}\left(\mathbf{A}^{\mathrm{T}} \mathbf{v}\right)^{\mathrm{T}} \boldsymbol{\Phi}\left(\mathbf{A}^{\mathrm{T}} \mathbf{v}\right) \mathrm{d} v
$$

Through Equation (C.3), the Appendix A, and the property of orthogonality of the modal functions, the relation $\mathbf{B}(t)^{\mathrm{T}} \mathbf{B}(t)=\mathbf{I}$ is found and consequently $\mathbf{B}(t)$ is an orthogonal matrix.

\section{Appendix D}

Let us consider different modes $n$-th and $m$-th and the corresponding modal functions $\boldsymbol{\Phi}^{<n>}$ and $\boldsymbol{\Phi}^{<m>}$. Equation (C.1) can be written for the $n$-th mode as follows

$$
\boldsymbol{\Phi}(\mathbf{v}) \mathbf{B}^{<n>}=\mathbf{A} \boldsymbol{\Phi}\left(\mathbf{A}^{\mathrm{T}} \mathbf{v}\right)^{<n>}
$$

Now, the last equation is pre-multiplied by $\rho \boldsymbol{\Phi}^{<m>}$, and then integrated over the solid volume, giving

$$
B_{m n}=\int_{\text {Volume }} \rho\left(\boldsymbol{\Phi}^{<m>}\right)^{\mathrm{T}} \mathbf{A} \boldsymbol{\Phi}\left(\mathbf{A}^{\mathrm{T}} \mathbf{v}\right)^{<n>} d v
$$


If the modes $\boldsymbol{\Phi}^{<n>}$ and $\boldsymbol{\Phi}^{<m>}$ are orthogonal, due to the geometry of revolution $\mathbf{A} \boldsymbol{\Phi}\left(\mathbf{A}^{\mathrm{T}} \mathbf{v}\right)^{<n>}$ and $\boldsymbol{\Phi}^{<m>}$ are orthogonal, and consequently $B_{m n}=0$.

The rows and columns of matrix $\mathbf{B}(t)$ associated with modes with multiplicity 1 have null entries except the diagonal entry, which is 1 . Due to the orthogonal property of $\mathbf{B}(t)$ (Appendix $\mathrm{C}$ ), the diagonal terms associated with modes with multiplicity 2 contain rotation matrices, that is

\section{Appendix E}

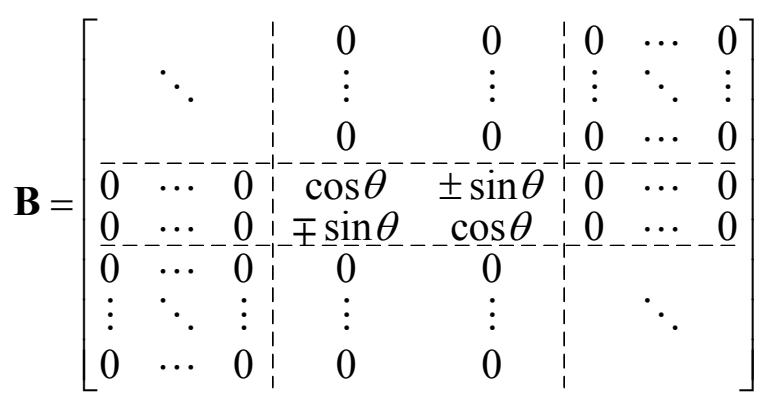

Let $\tilde{\mathbf{M}}$ be a diagonal matrix of dimension $N \times N$, where $N$ is the number of degrees of freedom of the solid model. The following relationship

$$
\mathbf{B}(t)^{\mathrm{T}} \tilde{\mathbf{M}} \mathbf{B}(t)=\tilde{\mathbf{M}}
$$

is verified if the pair of entries associated with each mode with multiplicity 2 are equal. The proof is found through the structure of the matrix $\mathbf{B}(t)$ showed in the Appendix D.

If $\tilde{\mathbf{M}}$ is a column matrix of dimension $N \times 1$, the following relation is fulfilled

$$
\mathbf{B}(t)^{\mathrm{T}} \tilde{\mathbf{M}}=\tilde{\mathbf{M}}
$$

only if the non-zero entries in $\widetilde{\mathbf{M}}$ are associated with modes of single multiplicity. 